\title{
Infinite Horizon Optimal Control of Stochastic Delay Evolution Equations in Hilbert Spaces
}

\author{
Xueping Zhu' and Jianjun Zhou ${ }^{2}$ \\ ${ }^{1}$ School of Astronautics, Northwestern Polytechnical University, Xian, Shaanxi 710072, China \\ ${ }^{2}$ College of Science, Northwest A\&F University, Yangling, Shaanxi 712100, China \\ Correspondence should be addressed to Jianjun Zhou; zhoujj198310@163.com
}

Received 6 September 2012; Accepted 16 December 2012

Academic Editor: Juan J. Trujillo

Copyright (c) $2013 \mathrm{X}$. Zhu and J. Zhou. This is an open access article distributed under the Creative Commons Attribution License, which permits unrestricted use, distribution, and reproduction in any medium, provided the original work is properly cited.

\begin{abstract}
The aim of the present paper is to study an infinite horizon optimal control problem in which the controlled state dynamics is governed by a stochastic delay evolution equation in Hilbert spaces. The existence and uniqueness of the optimal control are obtained by means of associated infinite horizon backward stochastic differential equations without assuming the Gâteaux differentiability of the drift coefficient and the diffusion coefficient. An optimal control problem of stochastic delay partial differential equations is also given as an example to illustrate our results.
\end{abstract}

\section{Introduction}

In this paper, we consider a controlled stochastic evolution equation of the following form:

$$
\begin{gathered}
d X^{u}(s)=A X^{u}(s) d s+F\left(s, X_{s}^{u}\right) d s \\
+G\left(s, X_{s}^{u}\right) R\left(s, X_{s}^{u}, u(s)\right) d s+G\left(s, X_{s}^{u}\right) d W(s), \\
s \geq t, \\
X_{t}^{u}=x,
\end{gathered}
$$

where

$$
X_{s}^{u}(l)=X^{u}(s+l), \quad l \in[-\tau, 0], x \in C([-\tau, 0], H) .
$$

$u$ is the control process in a measurable space $(U, \mathcal{U})$, and $W$ is a cylindrical Wiener process in a Hilbert space $\Xi$. $A$ is the generator of a strongly continuous semigroup of bounded linear operator in another Hilbert space $H$, and the coefficients $F$ and $G$, defined on $[0, \infty) \times C([-\tau, 0], H)$, are assumed to satisfy Lipschitz conditions with respect to appropriate norms. We introduce the cost function

$$
J(u)=E \int_{t}^{\infty} e^{-\lambda s} g\left(s, X_{s}^{u}, u(s)\right) d s .
$$

Here, $g$ is a given real function, $\lambda$ is large enough, and the control problem is understood in the weak sense. We wish to minimize the cost function over all admissible controls.

The particular form of the control system is essential for our results, but it covers numerous interesting cases. For example, in the particular cases $U=H$ and $R(t, x, u)=u$, the term $u(t) d t+d W(t)$ in the state equation can be considered as a control affected by noise.

The stochastic optimal control problem was considered in 1977 by Bismut [1]. The optimal control problem for stochastic partial differential equations in the framework of a compact control state space has been studied in [2-5]. Buckdahn and Raşcanu [6] considered an optimal control problem for a semilinear parabolic stochastic differential equation with a nonlinear diffusion coefficient, and the existence of a quasioptimal (nonrelaxed) control is showed without assuming convexity of the coefficients. In [7-11], the authors provided a direct (classical or mild) solution of the Hamilton-Jacobi-Bellman equation for the value function, which is then used to prove that the optimal control is related to the corresponding optimal trajectory by a feedback law. In Gozzi $[10,11]$, the existence and uniqueness of a mild solution of the associated Hamilton-Jacobi-Bellman equation are proved, when the diffusion term only satisfies weak nondegeneracy conditions. The proofs are based on 
the corresponding regularity properties of the transition semigroup of the associated Ornstein-Uhlenbeck process.

The main tools for the control problem are techniques from the theory of backward stochastic differential equations (BSDEs) in the sense of Pardoux and Peng, first considered in the nonlinear case in [12]; see [13,14] as general references. BSDEs have been successfully applied to control problems; see, for example, $[15,16]$ and we also refer the reader to [17-20]. Fuhrman and Tessiture [19] considered the optimal control problem for stochastic differential equation in the strong form, assuming Lipschitz conditions and allowing degeneracy of the diffusion coefficient, under some structural constraint on the state equation. Existence of an optimal control for stochastic systems in infinite dimensional spaces also has been obtained in [21-27]. In [21], Fuhrman and Tessitore showed the regularity with respect to parameters and the regularity in the Malliavin spaces for the solution of the backward-forward system and defined the feedback law by Malliavin calculus. Finally, the optimal control is obtained by the feedback. Appealing to the Malliavin calculus, compared with Fuhrman et al. [23], the existence of optimal control for stochastic differential equations with delay is proved by the feedback law. Fuhrman and Tessiture [24] dealt with an infinite horizon optimal control problem for the stochastic evolution equation in Hilbert space, and the optimal control is showed by means of infinite horizon backward stochastic differential equation in infinite dimensional spaces and Malliavin calculus. In Masiero [25], the infinite horizon optimal control problem for stochastic evolution equation is also studied by means of the Hamilton-Jacobi-Bellman equation. In Fuhrman [26], a class of optimal control problems governed by stochastic evolution equations in Hilbert spaces which includes state constraints is considered, and the optimal control is obtained by the Fleming logarithmic transformation. Masiero [27] studied stochastic evolution equations evolving in a Banach space where $G$ is a constant and characterized the optimal control via a feedback law by avoiding use of Malliavin calculus. Since there is a lack of regularity of $F$ and $G$, Malliavin calculus is not available in this case; the method in [27] also cannot be used as $G$ is not a constant, but we can prove a theorem similar to [26, Proposition 3.2], which will be used to define the feedback law.

In the present paper, we study the infinite horizon optimal control problem for stochastic delay evolution equations in Hilbert spaces, and by using Theorem 10, the optimal control is obtained. Since we do not relate the optimal feedback law with the gradient of the value function and do not consider the associated Hamilton-Jacobi-Bellman equation, we can drop the Gâteaux differentiability of the drift term and the diffusion term.

The plan of the paper is as follows. In the next section, some notations are fixed, and the stochastic delay evolution equations are considered with an infinite horizon; in particular, continuous dependence on initial value $(t, x)$ is proved. In Section 3, we give the proof of Theorem 10, which is the key of many subsequent results. The addressed optimal control problem is considered, and the fundamental relation between the optimal control problem and BSDEs is established in
Section 4. Section 5 is devoted to proving the existence and uniqueness of optimal control in the weak sense. Finally, an application is given in Section 6.

\section{Preliminaries}

We list some notations that are used in this paper. We use the symbol $|\cdot|$ to denote the norm in a Banach space $F$, with a subscript if necessary. Let $\Xi, H$, and $K$ denote real separable Hilbert spaces, with scalar products $(\cdot, \cdot)_{\Xi},(\cdot, \cdot)_{H}$, and $(\cdot, \cdot)_{K}$, respectively. For fixed $\tau>0, \mathscr{C}=C([-\tau, 0], H)$ denotes the space of continuous functions from $[-\tau, 0]$ to $H$, endowed with the usual norm $|f|_{C}=\sup _{\theta \in[-\tau, 0]}|f(\theta)|_{H}$. Let $\Xi^{*}$ denote the dual space of $\Xi$, with scalar product $(\cdot, \cdot)_{\Xi^{*}}$, and let $L(\Xi, H)$ denote the space of all bounded linear operators from $\Xi$ into $H$; the subspace of Hilbert-Schmidt operators, with the Hilbert-Schmidt norm, is denoted by $L_{2}(\Xi, H)$.

Let $(\Omega, \mathscr{F}, P)$ be a complete space with a filtration $\left\{\mathscr{F}_{t}\right\}_{t \geq 0}$ which satisfies the usual condition. By a cylindrical Wiener process with values in a Hilbert space $\Xi$, defined on $(\Omega, \mathscr{F}, P)$, we mean a family $\{W(t), t \geq 0\}$ of linear mappings $\Xi \rightarrow$ $L^{2}(\Omega)$ such that for every $\xi, \eta \in \Xi,\{W(t) \xi, t \geq 0\}$ is a real Wiener process and $E(W(t) \xi \cdot W(t) \eta)=(\xi, \eta)_{\Xi}$. In the following, $\{W(t), t \geq 0\}$ is a cylindrical Wiener process adapted to the filtration $\left\{\mathscr{F}_{t}\right\}_{t \geq 0}$.

In this section and the next section, $\left\{\mathscr{F}_{t}\right\}_{t \geq 0}$ will denote the natural filtration of $W$, augmented with the family $\mathcal{N}$ of $P$-null of $\mathscr{F}$. The filtration $\left\{\mathscr{F}_{t}, t \geq 0\right\}$ satisfies the usual conditions. For $[a, b],[a, \infty) \subset[0, \infty)$, we also use the following notations:

$$
\begin{gathered}
\mathscr{F}_{[a, b]}=\sigma(W(s)-W(a): s \in[a, b]) \vee \mathcal{N}, \\
\mathscr{F}_{[a, \infty)}=\sigma(W(s)-W(a): s \in[\mathrm{a}, \infty)) \vee \mathcal{N} .
\end{gathered}
$$

By $\mathscr{P}$ we denote the predictable $\sigma$-algebra, and by $\mathscr{B}(\Lambda)$ we denote the Borel $\sigma$-algebra of any topological space $\Lambda$.

Similar to [24], we define several classes of stochastic processes with values in a Banach space $F$ as follows.

(i) $L_{\mathscr{P}}^{2}(\Omega \times[t, \infty) ; F)$ denotes the space of equivalence classes of processes $Y \in L^{2}(\Omega \times[t, \infty) ; F)$, admitting a predictable version. $L_{\mathscr{P}}^{2}(\Omega \times[t, \infty) ; F)$ is endowed with the norm

$$
|Y|^{2}=E \int_{t}^{\infty}|Y(s)|^{2} d s .
$$

(ii) $L_{\mathscr{P}}^{p}\left(\Omega ; L_{\beta}^{q}([t, \infty) ; F)\right)$, defined for $\beta \in R$ and $p, q \in$ $[1, \infty)$, denotes the space of equivalence classes of processes $\{Y(s), s \geq t\}$, with values in $F$, such that the norm

$$
|Y|^{p}=E\left(\int_{t}^{\infty} e^{q \beta s}|Y(s)|^{q} d s\right)^{p / q}
$$

is finite and $Y$ admits a predictable version.

(iii) $\mathscr{K}_{\beta}^{p}(t)$ denotes the space $L_{\mathscr{P}}^{p}\left(\Omega ; L_{\beta}^{2}([t, \infty) ; F)\right) \times$ $L_{\mathscr{P}}^{p}\left(\Omega ; L_{\beta}^{2}\left([t, \infty) ; L_{2}(\Xi, F)\right)\right)$. The norm of an element 
$(Y, Z) \in \mathscr{K}_{\beta}^{p}$ is $|(Y, Z)|=|Y|+|Z|$. Here, $F$ is a Hilbert space.

(iv) $L_{\mathscr{P}}^{p}(\Omega ; C([t, T] ; F))$, defined for $T>t \geq 0$ and $p \in[1, \infty)$, denotes the space of predictable processes $\{Y(s), s \in[t, T]\}$ with continuous paths in $F$, such that the norm

$$
|Y|^{p}=E \sup _{s \in[t, T]}|Y(s)|^{p}
$$

is finite. Elements of $L_{\mathscr{P}}^{p}(\Omega ; C([t, T] ; F))$ are identified up to indistinguishability.

(v) $L_{\mathscr{P}}^{q}\left(\Omega ; C_{\eta}([t, \infty) ; F)\right)$, defined for $\eta \in R$ and $q \epsilon$ $[1, \infty)$, denotes the space of predictable processes $\{Y(s), s \geq t\}$ with continuous paths in $F$, such that the norm

$$
|Y|^{q}=E \sup _{s \geq t} e^{\eta q s}|Y(s)|^{q}
$$

is finite. Elements of $L_{\mathscr{P}}^{q}\left(\Omega ; C_{\eta}(F)\right)$ are identified up to indistinguishability.

(vi) Finally, for $\eta \in R$ and $q \in[1, \infty)$, we defined $\mathscr{H}_{\eta}^{\mathrm{q}}(t)$ as the space $L_{\mathscr{P}}^{q}\left(\Omega ; L_{\eta}^{q}([t, \infty) ; F)\right) \cap$ $L_{\mathscr{P}}^{q}\left(\Omega ; C_{\eta}([t, \infty) ; F)\right)$, endowed with the norm

$$
|Y|_{\mathscr{H}_{\eta}^{q}}=|Y|_{L_{\mathscr{P}}^{q}\left(\Omega ; L_{\eta}^{q}([t, \infty) ; F)\right)}+|Y|_{L_{\mathscr{P}}^{q}\left(\Omega ; C_{\eta}([t, \infty) ; F)\right)} .
$$

For simplicity, we denote $L_{\mathscr{P}}^{p}\left(\Omega ; L_{\beta}^{q}([0, \infty) ; F)\right), \quad L_{\mathscr{P}}^{q}(\Omega$; $\left.C_{\eta}([0, \infty) ; F)\right), \mathscr{H}_{\eta}^{q}(0)$, and $\mathscr{K}_{\beta}^{p}(0)$ by $L_{\mathscr{P}}^{p}\left(\Omega ; L_{\beta}^{q}(F)\right), L_{\mathscr{P}}^{q}(\Omega ;$ $\left.C_{\eta}(F)\right), \mathscr{H}_{\eta}^{q}$, and $\mathscr{K}_{\beta}^{p}$, respectively.

Now, for every fixed $t \geq 0$, we consider the following stochastic delay evolution equation:

$$
\begin{gathered}
d X(s)=A X(s) d s+F\left(t, X_{s}\right) d s+G\left(s, X_{s}\right) d W(s), \\
s \in[t, \infty), \\
X_{t}=x \in \mathscr{C} .
\end{gathered}
$$

We make the following assumptions.

Hypothesis 1. (i) The operator $A$ is the generator of a strongly continuous semigroup $\left\{e^{t A}, t \geq 0\right\}$ of bounded linear operators in the Hilbert space $H$. We denote by $M$ and $\omega$ two constants such that $\left|e^{t A}\right| \leq M e^{\omega t}$, for $t \geq 0$.

(ii) The mapping $F:[0, \infty) \times \mathscr{C} \rightarrow H$ is measurable and satisfies, for some constant $L>0$ and $0 \leq \theta<1$,

$$
\begin{gathered}
\left|e^{s A} F(t, x)\right| \leq L e^{\omega s} s^{-\theta}\left(1+|x|_{C}\right), \\
\left|e^{s A} F(t, x)-e^{s A} F(t, y)\right| \leq L e^{\omega s} s^{-\theta}|x-y|_{C} \\
s>0, t \in[0,+\infty), x, y \in \mathscr{C} .
\end{gathered}
$$

(iii) $G$ is a mapping $[0, \infty) \times \mathscr{C} \rightarrow L(\Xi, H)$ such that for every $v \in \Xi$, the map $G v$ : $[0, \infty) \times \mathscr{C} \rightarrow H$ is measurable, $e^{s A} G(t, x) \in L_{2}(\Xi, H)$ for every s $>0, t \in[0, \infty)$ and $x \in \mathscr{C}$, and

$$
\begin{gathered}
\left|e^{s A} G(t, x)\right|_{L_{2}(\Xi, H)} \leq L e^{\omega s} s^{-\gamma}\left(1+|x|_{C}\right), \\
\left|e^{s A} G(t, x)-e^{s A} G(t, y)\right|_{L_{2}(\Xi, H)} \leq L e^{\omega s} s^{-\gamma}\left(|x-y|_{C}\right), \\
s>0, \quad t \in[0,+\infty), \quad x, y \in \mathscr{C},
\end{gathered}
$$

for some constants $L>0$ and $\gamma \in[0,1 / 2)$.

We say that $X$ is a mild solution of (10) if it is a continuous, $\left\{\mathscr{F}_{t}\right\}_{t \geq 0}$-predictable process with values in $H$, and it satisfies $P$-a.s.,

$$
\begin{gathered}
X(s)=e^{(s-t) A} x(0)+\int_{t}^{s} e^{(s-\sigma) A} F\left(\sigma, X_{\sigma}\right) d \sigma \\
+\int_{t}^{s} e^{(s-\sigma) A} G\left(\sigma, X_{\sigma}\right) d W(\sigma), \quad s \in[t, \infty), \\
X_{t}=x \in \mathscr{C} .
\end{gathered}
$$

To stress dependence on initial data, we denote the solution by $X(s, t, x)$. Note that $X(s, t, x)$ is $\mathscr{F}_{[t, s]}$ measurable, hence, independent of $\mathscr{F}_{t}$.

We first recall a well-known result on solvability of (10) on bounded interval.

Theorem 1. Assume that Hypothesis 1 holds. Then, for all $q \in[2, \infty)$ and $T>0$, there exists a unique process $X \in$ $L_{\mathscr{P}}^{q}(\Omega, C([t, T] ; H))$ as mild solution of $(10)$. Moreover,

$$
E \sup _{s \in[t, T]}|X(s)|^{q} \leq C\left(1+|x|_{C}\right)^{q},
$$

for some constant $\mathrm{C}$ depending only on $q, \gamma, \theta, \mathrm{T}, \tau, \mathrm{L}, \omega$, and M.

By Theorem 1 and the arbitrariness of $T$ in its statement, the solution is defined for every $s \geq t$. We have the following result.

Theorem 2. Assume that Hypothesis 1 holds and the process $X(\cdot, t, x)$ is mild solution of (10) with initial value $(t, x) \in$ $[0, \infty) \times \mathscr{C}$. Then, for every $q \in[1, \infty)$, there exists a constant $\eta(q)$ such that the process $X .(t, x) \in \mathscr{H}_{\eta(q)}^{q}(t)$. Moreover, for a suitable constant $C>0$, one has

$$
\operatorname{Esup}_{s \geq t} e^{\eta(q) q s}\left|X_{s}\right|_{C}^{q}+E \int_{t}^{\infty} e^{\eta(q) q s}\left|X_{s}\right|_{C}^{q} d s \leq C\left(1+|x|_{C}\right)^{q},
$$

with the constant $\eta(q)$ depending only on $q, \gamma, \theta, \tau, L, \omega$, and M. 
Proof. We define a mapping $\Phi$ from $\mathscr{H}_{\eta}^{q}(t) \times[0, \infty) \times \mathscr{C}$ to $\mathscr{H}_{\eta}^{q}(t)$ by the formula

$$
\begin{gathered}
\Phi(X ., t, x)_{s}(l)=e^{(s+l-t) A} x(0)+\int_{t}^{s+l} e^{(s+l-\sigma) A} F\left(\sigma, X_{\sigma}\right) d \sigma \\
\quad+\int_{t}^{s+l} e^{(s+l-\sigma) A} G\left(\sigma, X_{\sigma}\right) d W(\sigma), \\
s \in[t, \infty), \quad l \in[-\tau, 0], \quad s+l \geq t, \\
\Phi(X ., t, x)_{s}(l)=x(s+l-t), \\
s \in[t, \infty), \quad l \in[-\tau, 0], \quad s+l<t .
\end{gathered}
$$

We are going to show that, provided $\eta$ is suitably chosen, $\Phi(\cdot, t, x)$ is well defined and that it is a contraction in $\mathscr{H}_{\eta}^{q}(t)$; that is, there exists $c<1$ such that

$$
\begin{aligned}
& \left|\Phi\left(X_{.}^{1}, t, x\right)-\Phi\left(X_{.}^{1}, t, x\right)\right|_{\mathscr{H}_{\eta}^{q}(t)} \\
& \quad \leq c\left|X_{.}^{1}-X_{\cdot}^{2}\right|_{\mathscr{H}_{\eta}^{q}(t)}, \quad X_{.}^{1}, X^{2} \in \mathscr{H}_{\eta}^{q}(t) .
\end{aligned}
$$

For simplicity, we set $t=0$, and we treat only the case $F=0$, the general case being handled in a similar way. We will use the so called factorization method; see [28, Theorem 5.2.5]. Let us take $q>1$ and $\alpha \in(0,1)$ such that $1 / q<\alpha<(1 / 2)-$ $\gamma, \quad$ and let $c_{\alpha}^{-1}=\int_{\sigma}^{s}(s-r)^{\alpha-1}(r-\sigma)^{-\alpha} d r$.

By the stochastic Fubini theorem,

$$
\begin{aligned}
& \Phi(X ., 0, x)_{s}(l)= e^{(s+l) A} x(0) \\
&+c_{\alpha} \int_{0}^{s+l} \int_{\sigma}^{s+l}(s+l-r)^{\alpha-1}(r-\sigma)^{-\alpha} \\
& \times e^{(s+l-r) A} e^{(r-\sigma) A} d r G\left(\sigma, X_{\sigma}\right) d W(\sigma) \\
&= e^{(s+l) A} x(0)+\Phi^{\prime}\left(X_{s}\right)(l), \\
& s \in[0, \infty), \quad l \in[-\tau, 0], \quad s+l \geq 0, \\
& \Phi(X, 0, x)_{s}(l)=x(s+l), \\
& s \in[0, \infty), \quad l \in[-\tau, 0], \quad s+l<0,
\end{aligned}
$$

where

$$
\begin{gathered}
\Phi^{\prime}(X .)_{s}(l)=c_{\alpha} \int_{0}^{s+l}(s+l-r)^{\alpha-1} e^{(s+l-r) A} Y(r) d r, \\
Y(r)=\int_{0}^{r}(r-\sigma)^{-\alpha} e^{(r-\sigma) A} G\left(\sigma, X_{\sigma}\right) d W(\sigma) .
\end{gathered}
$$

Since $\sup _{-\tau \leq l \leq 0}\left|e^{(s+l) A} x(0)\right| \leq M e^{\omega s}|x|_{C}$, the process $e^{(s+\cdot) A}$ $x(0), s \geq 0$, belongs to $\mathscr{H}_{\eta}^{q}$ provided $\omega+\eta<0$. Next, we estimate $\Phi^{\prime}(X$.$) , where$

$$
\left|\Phi^{\prime}(X .)_{s}(l)\right| \leq c_{\alpha} \int_{0}^{s+l}(s+l-r)^{\alpha-1} M e^{(s+l-r) \omega}|Y(r)| d r,
$$

setting $q^{\prime}=q /(q-1)$, so that

$$
\begin{aligned}
e^{q \eta s} \mid & \left.\Phi^{\prime}(X .)_{s}\right|^{q} \\
\leq & c_{\alpha}^{q} M^{q} \sup _{-\tau \leq l \leq 0} e^{q \eta s}\left(\int_{0}^{s+l}(s+l-r)^{\alpha-1} e^{\omega(s+l-r)}|Y(r)| d r\right)^{q} \\
\leq & c_{\alpha}^{q} M^{q} \sup _{-\tau \leq l \leq 0}\left(\int_{0}^{s+l}(s+l-r)^{\alpha-1} e^{\left((\omega+\eta) / q^{\prime}\right)(s+l-r)}\right. \\
\times & \left.\times e^{((\omega+\eta) / q)(s-r)} e^{\eta r}|Y(r)| d r\right)^{q} \\
\leq & c_{\alpha}^{q} M^{q} \sup _{-\tau \leq l \leq 0}\left(\int_{0}^{s+l} e^{(\eta+\omega)(s+l-r)}(s+l-r)^{(\alpha-1) q^{\prime}} d r\right)^{q / q^{\prime}} \\
& \times \int_{0}^{s+l} e^{(\eta+\omega)(s-r)} e^{q \eta r}|Y(r)|^{q} d r \\
\leq & c_{\alpha}^{q} M^{q}\left(\int_{0}^{s} e^{(\eta+\omega) r} r^{q^{\prime}(\alpha-1)} d r\right)^{q / q^{\prime}} \\
& \times \int_{0}^{s} e^{(\eta+\omega)(s-r)} e^{q \eta r}|Y(r)|^{q} d r .
\end{aligned}
$$

Applying the Young inequality for convolutions, we have

$$
\begin{aligned}
\int_{0}^{\infty} e^{q \eta s}\left|\Phi^{\prime}(X .)_{s}\right|^{q} d s \leq & c_{\alpha}^{q} M^{q}\left(\int_{0}^{\infty} e^{(\eta+\omega) s} s^{q^{\prime}(\alpha-1)} d s\right)^{q / q^{\prime}} \\
& \times \int_{0}^{\infty} e^{(\eta+\omega) s} d s \int_{0}^{\infty} e^{q \eta s}|Y(s)|^{q} d s
\end{aligned}
$$

and we conclude that

$$
\begin{aligned}
\left|\Phi^{\prime}(X .)\right|_{L_{\mathscr{P}}^{q}\left(\Omega ; L_{\eta}^{q}(\mathscr{C})\right)} \leq & c_{\alpha} M|Y|_{L_{\mathscr{P}}^{q}\left(\Omega ; L_{\eta}^{q}(H)\right)} \\
& \times\left(\int_{0}^{\infty} e^{(\eta+\omega) s} s^{q^{\prime}(\alpha-1)} d s\right)^{1 / q^{\prime}} \\
& \times\left(\int_{0}^{\infty} e^{(\eta+\omega) s} d s\right)^{1 / q} .
\end{aligned}
$$

If we start again from (20) and apply the Hölder inequality, we obtain

$$
\begin{aligned}
\left|e^{\eta(s+l)} \Phi^{\prime}(X .)_{s}(l)\right| \leq & c_{\alpha} M\left(\int_{0}^{s+l} r^{(\alpha-1) q^{\prime}} e^{(\omega+\eta) r q^{\prime}} d r\right)^{1 / q^{\prime}} \\
& \times\left(\int_{0}^{s+l} e^{\eta r q}|Y(r)|^{q} d r\right)^{1 / q}, \\
\left|e^{\eta s} \Phi^{\prime}(X .)_{s}\right| \leq & c_{\alpha} M\left(\int_{0}^{s} r^{(\alpha-1) q^{\prime}} e^{(\omega+\eta) r q^{\prime}} d r\right)^{1 / q^{\prime}} \\
& \times\left(\int_{0}^{s} e^{\eta r q}|Y(r)|^{q} d r\right)^{1 / q} .
\end{aligned}
$$


So, we conclude that

$$
\begin{aligned}
\left|\Phi^{\prime}(X .)\right|_{L_{\mathscr{P}}^{q}\left(\Omega ; C_{\eta}(\mathscr{C})\right)} \leq & \mathcal{c}_{\alpha} M|Y|_{L_{\mathscr{P}}^{q}\left(\Omega ; L_{\eta}^{q}(H)\right)} \\
& \times\left(\int_{0}^{\infty} r^{(\alpha-1) q^{\prime}} e^{(\omega+\eta) r q^{\prime}} d r\right)^{1 / q^{\prime}} .
\end{aligned}
$$

On the other hand, by the Burkholder-Davis-Gundy inequalities, for some constant $c_{q}$ depending only on $q$, we have

$$
\begin{aligned}
E|Y(r)|^{q} \leq & c_{q} E\left(\int_{0}^{r}(r-\sigma)^{-2 \alpha}\left|e^{(r-\sigma) A} G\left(\sigma, X_{\sigma}\right)\right|_{L_{2}(\Xi, H)}^{2} d \sigma\right)^{q / 2} \\
\leq & L^{q} c_{q} E \\
& \times\left(\int_{0}^{r}(r-\sigma)^{-2 \alpha-2 \gamma} e^{2 \omega(r-\sigma)}\left(1+\left|X_{\sigma}\right|_{C}^{2}\right) d \sigma\right)^{q / 2},
\end{aligned}
$$

which implies that

$$
\begin{aligned}
{\left[E|Y(r)|^{q}\right]^{2 / q} \leq } & L^{2} c_{q}^{2 / q} \int_{0}^{r}(r-\sigma)^{-2 \alpha-2 \gamma} \\
& \times e^{2 \omega(r-\sigma)}\left[E\left(1+\left|X_{\sigma}\right|_{C}\right)^{q}\right]^{2 / q} d \sigma,
\end{aligned}
$$

so that

$$
\begin{aligned}
e^{2 \eta r}\left[E|Y(r)|^{q}\right]^{2 / q} \leq & C_{1} \int_{0}^{r}(r-\sigma)^{-2 \alpha-2 \gamma} e^{2(\omega+\eta)(r-\sigma)} e^{2 \eta \sigma} d \sigma \\
& +C_{2} \int_{0}^{r}(r-\sigma)^{-2 \alpha-2 \gamma} e^{2(\omega+\eta)(r-\sigma)} \\
& \times e^{2 \eta \sigma}\left[E\left|X_{\sigma}\right|_{C}^{q}\right]^{2 / q} d \sigma,
\end{aligned}
$$

for suitable constants $C_{1}, C_{2}$. Applying the Young inequality for convolutions, we obtain

$$
\begin{aligned}
\int_{0}^{\infty} e^{q \eta r} E|Y(r)|^{q} d s \leq & C_{1}\left(\int_{0}^{\infty} s^{-2 \alpha-2 \gamma} e^{2(\omega+\eta) s} d s\right)^{q / 2} \int_{0}^{\infty} e^{q \eta s} d s \\
& +C_{2}\left(\int_{0}^{\infty} s^{-2 \alpha-2 \gamma} e^{2(\omega+\eta) s} d s\right)^{q / 2} \\
& \times \int_{0}^{\infty} e^{q \eta s} E\left|X_{s}\right|_{C}^{q} d s .
\end{aligned}
$$

This shows that $|Y|_{L_{\mathscr{P}}^{q}\left(\Omega ; L_{\eta}^{q}(H)\right)}$ is finite provided we assume that $\eta<0$ and $\omega+\eta<0$; so, the map is well defined.

If $X^{1}, X^{2}$. are processes belonging to $\mathscr{H}_{\eta}^{q}$ and $Y^{1}, Y^{2}$ are defined accordingly, the entirely analogous passages show that

$$
\begin{aligned}
\left|Y^{1}-Y^{2}\right|_{L_{\mathscr{P}}^{q}\left(\Omega ; L_{\eta}^{q}(H)\right)} \leq & L c_{\alpha}^{1 / q}\left|X^{1}-X_{.}^{2}\right|_{L_{\mathscr{P}}^{q}\left(\Omega ; L_{\eta}^{q}(\mathscr{C})\right)} \\
& \times\left(\int_{0}^{\infty} s^{-2 \alpha-2 \gamma} e^{2(\omega+\eta) s} d s\right)^{1 / 2} .
\end{aligned}
$$

Recalling the inequalities (23) and (25) and noting that the map $\mathrm{Y} \rightarrow \Phi^{\prime}(\mathrm{X}$.$) is linear, we obtain an explicit expression$ for the constant $c$ in (17), and it is immediate to verify that $c<1$ provided $\eta<0$ is chosen sufficiently large. We fix such a value of $\eta(q)$. The first result is a consequence of the contraction principle. The estimate (15) also follows from the contraction property of $\Phi(\cdot, t, x)$.

For investigating the dependence of the solution $X(s, t, x)$ on the initial data $x$ and $t$, we reformulate (13) as an equation on $[0, \infty)$. We set

$$
S(s)=e^{s A}, \quad \text { for } s \geq 0, \quad S(s)=I, \quad \text { for } s<0,
$$

and we consider the equation

$$
X(s)=S(s-t) x((0 \wedge(s-t)) \vee(-\tau))
$$

$$
\begin{aligned}
& +\int_{0}^{s} I_{[t, \infty)}(\sigma) S(s-\sigma) F\left(\sigma, X_{\sigma}\right) d \sigma \\
& +\int_{0}^{s} I_{[t, \infty)}(\sigma) S(s-\sigma)
\end{aligned}
$$

$$
\begin{gathered}
\times G\left(\sigma, X_{\sigma}\right) d W(\sigma), \quad s \in[0, \infty), \\
X_{0}(\theta)=x((-t+\theta) \vee(-\tau)), \quad \theta \in[-\tau, 0] .
\end{gathered}
$$

Under the assumptions of Hypothesis 1, by Theorem 2, it is easy to prove that equation (32) has a unique solution $X$ and $X . \in \mathscr{H}_{\eta(q)}^{q}$ for every $q \in[2, \infty)$. It clearly satisfies $X(s)=$ $x((s-t) \vee(-\tau))$ for $s \in[-\tau, t)$, and its restriction to the time internal $[t, \infty)$ is the unique mild solution of (10). From now on, we denote by $X(s, t, x), s \in[0, \infty)$, the solution of (32).

We need the following parameter-depending contraction principle, which is stated in the following lemma and proved in [29, Theorems 10.1 and 10.2].

Lemma 3 (Parameter Depending Contraction Principle). Let $B, D$ denote Banach spaces. Let $h: B \times D \rightarrow B$ be a continuous mapping satisfying

$$
\left|h\left(x_{1}, y\right)-h\left(x_{2}, y\right)\right| \leq \alpha\left|x_{1}-x_{2}\right|
$$

for some $\alpha \in[0,1)$ and every $x_{1}, x_{2} \in B, y \in D$. Let $\phi(y)$ denote the unique fixed point of the mapping $h(\cdot, y): B \rightarrow B$. Then, $\phi: D \rightarrow B$ is continuous.

Theorem 4. Assume that Hypothesis 1 holds true. Then, for every $q \in[1, \infty)$, the map $(t, x) \rightarrow X .(t, x)$ is continuous from $[0, \infty) \times \mathscr{C}$ to $\mathscr{H}_{\eta(q)}^{q}$.

Proof. Clearly, it is enough to prove the claim for $q$ large. Let us consider the map $\Phi$ defined in the proof of Theorem 2. In 
our present notation, $\Phi$ can be seen as a mapping from $\mathscr{H}_{\eta}^{q} \times$ $[0, \infty) \times \mathscr{C}$ to $\mathscr{H}_{\eta}^{q}$ as follows:

$$
\begin{gathered}
\Phi(X ., t, x)_{s}(l)=S(s+l-t) x(0) \\
\quad+\int_{0}^{s+l} I_{[t, \infty)}(\sigma) S(s+l-\sigma) F\left(\sigma, X_{\sigma}\right) d \sigma \\
\quad+\int_{0}^{s+l} I_{[t, \infty)}(\sigma) S(s+l-\sigma) \\
\quad \times G\left(\sigma, X_{\sigma}\right) d W(\sigma), \\
s \in[0, \infty), \quad l \in[-\tau, 0], \quad s+l \geq t \\
\Phi(X, t, x)_{s}(l)=x((s+l-t) \vee(-\tau)) \\
s \in[0, \infty), \quad l \in[-\tau, 0], \quad s+l \leq t .
\end{gathered}
$$

By the arguments of the proof of Theorem 2, $\Phi(\cdot, t, x)$ is a contraction in $\mathscr{H}_{\eta}^{q}$ uniformly with respect to $t, x$. The process $X .(t, x)$ is the unique fixed point of $\Phi(\cdot, t, x)$. So, by the parameter-depending contraction principle (Lemma 3), it suffices to show that $\Phi$ is continuous from $\mathscr{H}_{\eta}^{q} \times[0, \infty) \times \mathscr{C}$ to $\mathscr{H}_{\eta}^{q}$. From the contraction property of $\Phi(\cdot, t, x)$ mentioned earlier, we have that $\Phi(\cdot, t, x)$ is continuous, uniformly in $t, x$. Moreover, for fixed $X$., it is easy to verify that $\Phi\left(X_{.}, \cdot, \cdot\right)$ is continuous from $[0, \infty) \times \mathscr{C}$ to $\mathscr{H}_{\eta}^{q}$. The proof is finished.

Remark 5. By similar passages, we can show that, for fixed $t$, Theorem 4 still holds true for $q$ large enough if the spaces $[0, \infty) \times \mathscr{C}$ and $\mathscr{H}_{\eta}^{q}$ are replaced by the spaces $L^{q}\left(\Omega, \mathscr{C}, \mathscr{F}_{t}\right)$ and $\mathscr{H}_{\eta}^{q}(t)$ respectively, where $L^{q}\left(\Omega, \mathscr{C}, \mathscr{F}_{t}\right)$ denotes that the space of $\mathscr{F}_{t}$-measurable function with value in $\mathscr{C}$, such that the norm

$$
|x|^{q}=E|x|_{C}^{q}
$$

is finite.

\section{The Backward-Forward System}

In this section, we consider the system of stochastic differential equations, $P$-a.s.,

$$
\begin{gathered}
X(s)=e^{(s-t) A} x(0)+\int_{t}^{s} e^{(s-\sigma) A} F\left(\sigma, X_{\sigma}\right) d \sigma \\
\quad+\int_{t}^{s} e^{(s-\sigma) A} G\left(\sigma, X_{\sigma}\right) d W(\sigma), \quad s \in[t, \infty), \\
X_{t}=x \in \mathscr{C}, \\
Y(s)-Y(T)+\int_{s}^{T} Z(\sigma) d W(\sigma)+\lambda \int_{s}^{T} Y(\sigma) d \sigma \\
=\int_{s}^{T} \psi\left(\sigma, X_{\sigma}, Y(\sigma), Z(\sigma)\right) d \sigma, \quad 0 \leq s \leq T<\infty,
\end{gathered}
$$

for $s$ varying on the time interval $[t, \infty) \subset[0, \infty)$. As in Section 2, we extend the domain of the solution setting $X(s, t, x)=x((s-t) \vee(-\tau))$ for $s \in[-\tau, t)$.

We make the following assumptions.

Hypothesis 2. The mapping $\psi:[0, \infty) \times \mathscr{C} \times K \times L_{2}(\Xi, K) \rightarrow$ $K$ is Borel measurable such that, for all $t \in[0, \infty), \psi(t, \cdot): \mathscr{C} \times$ $K \times L_{2}(\Xi, K) \rightarrow K$ is continuous, and for some $L_{y}, L_{z}>0$, $\mu \in R$, and $m \geq 1$,

$$
\begin{gathered}
\left|\psi\left(s, x, y_{1}, z_{1}\right)-\psi\left(s, x, y_{2}, z_{2}\right)\right| \\
\leq L_{y}\left|y_{1}-y_{2}\right|+L_{z}\left|z_{1}-z_{2}\right|, \\
|\psi(s, x, y, z)| \leq L\left(1+|x|_{C}^{m}+|y|+|z|\right), \\
\left\langle\psi\left(s, x, y_{1}, z\right)-\psi\left(s, x, y_{2}, z\right), y_{1}-y_{2}\right\rangle_{K} \geq \mu\left|y_{1}-y_{2}\right|^{2},
\end{gathered}
$$

for every $s \in[0, \infty), x \in \mathscr{C}, y, y_{1}, y_{2} \in K, z, z_{1}$, and $z_{2} \in$ $L_{2}(\Xi, K)$.

We note that the third inequality in (37) follows from the first one taking $\mu=-L_{y}$ but that the third inequality may also hold for different values of $\mu$.

Firstly, we consider the backward stochastic differential equation

$$
\begin{aligned}
Y(s) & -Y(T)+\int_{s}^{T} Z(\sigma) d W(\sigma)+\lambda \int_{s}^{T} Y(\sigma) d \sigma \\
& =\int_{s}^{T} \psi\left(\sigma, X_{\sigma}, Y(\sigma), Z(\sigma)\right) d \sigma, \quad 0 \leq s \leq T<\infty .
\end{aligned}
$$

$K$ is a Hilbert space, the mapping $\psi:[0, \infty) \times \mathscr{C} \times K \times$ $L_{2}(\Xi, K) \rightarrow K$ is a given measurable function, $X$. is a predictable process with values in another Banach space $\mathscr{C}$, and $\lambda$ is a real number.

Theorem 6. Assume that Hypothesis 2 holds. Let $p>2$ and $\delta<0$ be given, and choose

$$
q \geq m p, \quad \eta>\frac{\delta}{m} .
$$

Then, the following hold.

(i) For $X . \in L_{\mathscr{P}}^{q}\left(\Omega ; L_{\eta}^{q}(\mathscr{C})\right)$ and $\lambda>-\left(\delta+\mu-\left(L_{z}^{2} / 2\right)\right)$, (38) has a unique solution in $\mathscr{K}_{\delta}^{p}$ that will be denoted by $(Y(X).(s), Z(X).(s)), s \geq 0$.

(ii) The estimate

$$
\begin{aligned}
\operatorname{Eup}_{s \geq 0}( & Y(X .)(s))^{p} e^{p \delta s}+E\left(\int_{0}^{\infty} e^{2 \delta \sigma}|Y(X .)(\sigma)|^{2} d \sigma\right)^{p / 2} \\
& +E\left(\int_{0}^{\infty} e^{2 \delta \sigma}|Z(X .)(\sigma)|^{2} d \sigma\right)^{p / 2} \\
\leq & c\left(1+|X|_{L_{\mathscr{P}}^{q}\left(\Omega ; L_{\eta}^{q}(\mathscr{C})\right)}^{m}\right)^{p}
\end{aligned}
$$


holds for a suitable constant c. In particular, $Y(X.) \in$ $L_{\mathscr{P}}^{p}\left(\Omega ; C_{\delta}(K)\right)$.

(iii) The map $X . \rightarrow(Y(X),. Z(X)$.$) is continuous from$ $L_{\mathscr{P}}^{q}\left(\Omega ; L_{\eta}^{q}(\mathscr{C})\right)$ to $\mathscr{K}_{\delta}^{p}$, and $X . \rightarrow Y(X$.$) is continuous$ from $L_{\mathscr{P}}^{q}\left(\Omega ; L_{\eta}^{q}(\mathscr{C})\right)$ to $L_{\mathscr{P}}^{p}\left(\Omega ; C_{\delta}(K)\right)$.

(iv) The statements of points (i), (ii), and (iii) still hold true if the space $L_{\mathscr{P}}^{q}\left(\Omega ; L_{\eta}^{q}(\mathscr{C})\right)$ is replaced by the space $L_{\mathscr{P}}^{q}\left(\Omega ; C_{\eta}(\mathscr{C})\right)$.

Proof. The theorem is very similar to Proposition 3.11 in [24]. The only minor difference is that the mapping $\psi:[0, \infty) \times$ $\mathscr{C} \times K \times L_{2}(\Xi, K) \rightarrow K$ is a given measurable function, while in [24], the measurable function $\psi$ is from $H \times K \times L_{2}(\Xi, K)$ to $K$; however, the same arguments apply.

Theorem 7. Assume that Hypothesis 1 holds and that Hypothesis 2 holds true in the particular case $K=R$. Then, for every $p>2, q, \delta<0$ satisfying (39) with $\eta=\eta(q)$, and for every $\lambda>\lambda^{\prime}=-\left(\delta+\mu-\left(L_{z}^{2} / 2\right)\right)$, there exists a unique solution in $\mathscr{H}_{\eta(q)}^{q} \times \mathscr{K}_{\delta}^{p}$ of (36) that will be denoted by $(X(\cdot, t, x), Y(\cdot, t, x), Z(\cdot, t, x))$. Moreover, $Y(\cdot, t, x) \quad \in$ $L_{\mathscr{P}}^{p}\left(\Omega ; C_{\delta}(R)\right)$. The map $(t, x) \rightarrow(Y(\cdot, t, x), Z(\cdot, t, x))$ is continuous from $[0, \infty) \times \mathscr{C}$ to $\mathscr{K}_{\delta}^{p}$, and the map $(t, x) \rightarrow Y(\cdot, t, x)$ is continuous from $[0, \infty) \times \mathscr{C}$ to $L_{\mathscr{P}}^{p}\left(\Omega ; C_{\delta}(R)\right)$.

Proof. We first notice that the system is decoupled; the first does not contain the solution $(Y, Z)$ of the second one. Therefore, under the assumption of Hypothesis 1 by Theorem 2, there exists a unique solution $X(\cdot, t, x)$ and $X .(t, x) \in \mathscr{H}_{\eta(q)}^{q}$ of the first equation. Moreover, from Theorem 4, it follows that the map $(t, x) \rightarrow X .(t, x)$ is continuous from $[0, \infty) \times \mathscr{C}$ to $\mathscr{H}_{\eta(q)}^{q}$.

Let $K=R$; from Theorem 6, we have that there exists a unique solution $(Y(\cdot, t, x), Z(\cdot, t, x)) \in \mathscr{K}_{\delta}^{p}$ of the second equation, and the map $X \rightarrow(Y(X),. Z(X)$.$) is$ continuous from $\mathscr{H}_{\eta(q)}^{q}$ to $\mathscr{K}_{\delta}^{p}$ while $\mathrm{X} \rightarrow(\mathrm{Y}(\mathrm{X})$.$) is$ continuous from $\mathscr{H}_{\eta(q)}^{q}$ to $L_{\mathscr{P}}^{p}\left(\Omega ; C_{\delta}(R)\right)$. We have proved that $(X(\cdot, t, x), Y(\cdot, t, x), Z(\cdot, t, x)) \in \mathscr{H}_{\eta(q)}^{q} \times \mathscr{K}_{\delta}^{p}$ is the unique solution of (36), and the other assertions follow from composition.

Remark 8. From Remark 5, by similar passages, we can show that for fixed $t$ and for $q$ large enough, under the assumptions of Theorem 7 , the map $x \rightarrow(Y(\cdot, t, x), Z(\cdot, t, x))$ is continuous from $L^{q}\left(\Omega, \mathscr{C}, \mathscr{F}_{t}\right)$ to $\mathscr{K}_{\delta}^{p}(t)$.

We also remark that the process $X(\cdot, t, x)$ is $\mathscr{F}_{[t, \infty)}$ measurable, since $\mathscr{C}$ is separable Banach space, we have that $X .(t, x)$ is $\mathscr{F}_{[t, \infty)}$ measurable; So that $Y(t)$ is measurable with respect to both $\mathscr{F}_{[t, \infty)}$ and $\mathscr{F}_{t}$, it follows that $Y(t)$ is deterministic.

For later use, we notice three useful identities; for $t \leq s<$ $\infty$, the equality, $P$-a.s.,

$$
X_{l}\left(s, X_{s}(t, x)\right)=X_{l}(t, x), \quad l \in[s, \infty)
$$

is a consequence of the uniqueness of the solution of (13). Since the solution of the backward equation is uniquely determined on an interval $[s, \infty)$ by the values of the process $X$. on the same interval, for $t \leq s<\infty$, we have, $P$-a.s.,

$$
\begin{gathered}
Y\left(l, s, X_{s}(t, x)\right)=Y(l, t, x), \quad \text { for } l \in[s, \infty), \\
Z\left(l, s, X_{s}(t, x)\right)=Z(l, t, x), \quad \text { for a.a. } l \in[s, \infty) .
\end{gathered}
$$

Lemma 9 (see [30]). Let $E$ be a metric space with metric $d$, and let $f: \Omega \rightarrow E$ be strongly measurable. Then, there exists a sequence $f_{n}, n \in N$, of simple E-valued functions (i.e., $f_{n}$ is $\mathscr{F} / \mathscr{B}(E)$ measurable and takes only a finite number of values) such that for arbitrary $\omega \in \Omega$, the sequence $d\left(f_{n}(\omega), f(\omega)\right), n \in N$, is monotonically decreasing to zero.

Let now $f \in L^{q}(\Omega, \mathscr{C})$. By Lemma 9 we get the existence of a sequence of simple function $f_{n}, n \in N$, such that

$$
\left|f_{n}(\omega)-f(\omega)\right| \downarrow 0 \quad \text { for all } \omega \in \Omega \text { as } n \rightarrow \infty \text {. }
$$

Hence, $f_{n} \rightarrow f$ in $|\cdot|_{L^{q}(\Omega, \mathscr{C})}$ by Lebesgue's dominated convergence theorem.

We are now in a position of showing the main result in this section.

Theorem 10. Assume that Hypothesis 1 holds true and that Hypothesis 2 holds in the particular case $K=R$. Then, there exist two Borel measurable deterministic functions $v:[t, \infty) \times$ $\mathscr{C} \rightarrow R$ and $\zeta:[t, \infty) \times \mathscr{C} \rightarrow \Xi^{*}=L(\Xi, R)=$ $L_{2}(\Xi, R)$, such that for $t \in[0, \infty)$ and $x \in \mathscr{C}$, the solution $(X(t, x), Y(t, x), Z(t, x))$ of $(36)$ satisfies

$$
\begin{array}{r}
Y(s, t, x)=v\left(s, X_{s}(t, x)\right), \quad Z(s, t, x)=\zeta\left(s, X_{s}(t, x)\right), \\
P \text {-a.s., for a.a. } s \in[t, \infty)
\end{array}
$$

Proof. We apply the techniques introduced in [26, Proposition 3.2]. Let $\left\{e_{i}\right\}$ be a basis of $\Xi^{*}$, and let us define $Z^{i, N}=$ $\left(\left(Z, e_{i}\right)_{\Xi^{*}} \wedge N\right) \vee(-N)$. Then, for every $\left.0 \leq t_{1}<t_{2}<\infty, \Delta\right\rangle$ 0 , and $x_{1}, x_{2} \in \mathscr{C}$, we have that

$$
\begin{aligned}
& \left|E \int_{t_{1}}^{t_{1}+\Delta} Z^{i, N}\left(s, t_{1}, x_{1}\right) d s-E \int_{t_{2}}^{t_{2}+\Delta} Z^{i, N}\left(s, t_{2}, x_{2}\right) d s\right| \\
& \leq E \int_{t_{1}}^{t_{2}}\left|Z^{i, N}\left(s, t_{1}, x_{1}\right)\right| d s \\
& \quad+E \int_{t_{2}}^{t_{1}+\Delta}\left|Z^{i, N}\left(s, t_{1}, x_{1}\right)-Z^{i, N}\left(s, t_{2}, x_{2}\right)\right| d s \\
& \quad+E \int_{t_{1}+\Delta}^{t_{2}+\Delta}\left|Z^{i, N}\left(s, t_{2}, x_{2}\right)\right| d s
\end{aligned}
$$




$$
\begin{aligned}
\leq & 2 N\left|t_{2}-t_{1}\right|+\Delta^{1 / 2} e^{-\delta\left(t_{1}+\Delta\right)} \\
& \times\left(E\left(\int_{0}^{\infty} e^{2 \delta s}\left|Z^{i, N}\left(s, t_{1}, x_{1}\right)-Z^{i, N}\left(s, t_{2}, x_{2}\right)\right|^{2} d s\right)^{p / 2}\right)^{1 / p} \\
\leq & 2 N\left|t_{2}-t_{1}\right|+\Delta^{1 / 2} e^{-\delta\left(t_{1}+\Delta\right)} \\
& \times\left(E\left(\int_{0}^{\infty} e^{2 \delta s}\left|Z\left(s, t_{1}, x_{1}\right)-Z\left(s, t_{2}, x_{2}\right)\right|^{2} d s\right)^{p / 2}\right)^{1 / p} .
\end{aligned}
$$

From Theorem 7, we have that the map $(t, x) \rightarrow \int_{t}^{t+\Delta}$ $Z^{i, N}(s, t, x) d s$ is continuous from $[0, \infty) \times \mathscr{C}$ to $R$. By Remark 8, we also have that, for fixed $t$, the map $x \rightarrow$ $E \int_{t}^{t+\Delta} E Z^{i, N}(s, t, x) d s$ is continuous from $L^{q}\left(\Omega, \mathscr{C}, \mathscr{F}_{t}\right)$ to $R$ for $q$ large enough. Let us define

$$
\begin{array}{r}
\zeta^{i, N}(t, x)=\liminf _{n \rightarrow \infty} n E \int_{t}^{t+(1 / n)} Z^{i, N}(s, t, x) d s \\
t \in[0, \infty), x \in \mathscr{C} .
\end{array}
$$

It is clear that $\zeta^{i, N}:[0, \infty) \times \mathscr{C} \rightarrow R$ is a Borel function.

We fix $x$ and $0 \leq t \leq s<\infty$. For $l \in[s, \infty)$, we denote $\left.E\left[Z^{i, N}(l, s, y)\right]\right|_{y=X_{s}(t, x)}$, the random variable obtained by composing $X_{s}(t, x)$ with the map $y \rightarrow E\left[Z^{i, N}(l, s, y)\right]$.

By Lemma 9 , there exists a sequence of $\mathscr{C}$-valued $\mathscr{F}_{s}{ }^{-}$ measurable simple functions

$$
f_{m}: \Omega \longrightarrow \mathscr{C}, f_{m}=\sum_{k=1}^{N_{m}} h_{k}^{(m)} I_{\left\{f_{m}=h_{k}^{(m)}\right\}}, \quad N_{m} \in N
$$

where $h_{1}^{(m)}, \ldots, h_{m}^{(m)}$ are pairwise distinct and $\Omega=\bigcup_{k=1}^{N_{m}}\left\{f_{m}=\right.$ $\left.h_{k}^{(m)}\right\}$, such that

$$
\left|f_{m}(\omega)-X_{s}(\omega)\right| \downarrow 0 \quad \text { for all } \omega \in \Omega \text { as } n \longrightarrow \infty \text {. }
$$

For any $B \in \mathscr{F}_{s}$, we have

$$
\begin{aligned}
\int_{B} \int_{s}^{s+(1 / n)} Z^{i, N}(l, t, x) d l d P \\
\quad=\int_{B} \int_{s}^{s+(1 / n)} Z^{i, N}\left(l, s, X_{s}\right) d l d P \\
\quad=E I_{B} \int_{s}^{s+(1 / n)} Z^{i, N}\left(l, s, X_{s}\right) d l \\
\quad=\lim _{m \rightarrow \infty} E\left(I_{B} \int_{s}^{s+(1 / n)} Z^{i, N}\left(l, s, f_{m}\right) d l\right)
\end{aligned}
$$

$$
\begin{aligned}
& =\lim _{m \rightarrow \infty} \sum_{k=1}^{N_{m}} E\left(I_{B} I_{\left\{f_{m}=h_{k}^{(m)}\right\}} \int_{s}^{s+(1 / n)} Z^{i, N}\left(l, s, h_{k}^{(m)}\right) d l\right) \\
& =\lim _{m \rightarrow \infty} E\left(I_{B} \sum_{k=1}^{N_{m}} I_{\left\{f_{m}=h_{k}^{(m)}\right\}}\right) E \int_{s}^{s+(1 / n)} Z^{i, N}\left(l, s, h_{k}^{(m)}\right) d l \\
& =\lim _{m \rightarrow \infty} E I_{B}\left(\left.E \int_{s}^{s+(1 / n)} Z^{i, N}(l, s, y) d l\right|_{y=f_{m}}\right) \\
& =\lim _{m \rightarrow \infty} \int_{B}\left(\left.E \int_{s}^{s+(1 / n)} Z^{i, N}(l, s, y) d l\right|_{y=f_{m}}\right) d P \\
& =\int_{B}\left(\left.E \int_{s}^{s+(1 / n)} Z^{i, N}(l, s, y) d l\right|_{y=X_{s}}\right) d P .
\end{aligned}
$$

and we get that

$$
\begin{aligned}
\zeta^{i, N}\left(s, X_{s}(t, x)\right)= & \liminf _{n \rightarrow \infty} n \\
& \times\left[\left.E \int_{s}^{s+(1 / n)} Z^{i, N}(l, s, y) d l\right|_{y=X_{s}(t, x)}\right] \\
& =\liminf _{n \rightarrow \infty} n E\left[\int_{s}^{s+(1 / n)} Z^{i, N}(l, t, x) d l \mid \mathscr{F}_{s}\right],
\end{aligned}
$$

Fix $t$ and $x$. Recalling that $\left|Z^{i, N}\right| \leq N$, by the Lebesgue theorem on differentiation, it follows that $P$-a.s.

$$
\begin{array}{r}
\lim _{n \rightarrow \infty} n \int_{s}^{s+(1 / n)} Z^{i, N}(l, t, x) d l=Z^{i, N}(s, t, x), \\
\text { for a.a. } s \in[t, \infty) .
\end{array}
$$

By the boundedness of $Z^{i, N}$, applying the dominated convergence theorem, we get that

$$
\begin{array}{r}
\zeta^{i, N}\left(s, X_{s}(t, x)\right)=E\left[Z^{i, N}(s, t, x) \mid \mathscr{F}_{s}\right]=Z^{i, N}(s, t, x), \\
P \text {-a.s., for a.a. } s \in[t, \infty) .
\end{array}
$$

Now, we have proved that for every $t, x$,

$$
\begin{array}{r}
\zeta^{i, N}\left(s, X_{s}(t, x)\right)=Z^{i, N}(s, t, x), \\
P \text {-a.s. for a.a. } s \in[t, \infty),
\end{array}
$$

for every $i, N$. Let $C \subset[0, \infty) \times \mathscr{C}$ denote the set of pairs $(t, x)$ such that $\lim _{N \rightarrow \infty} \zeta^{i, N}(t, x)$ exists and the series $\sum_{i=1}^{\infty}\left(\lim _{N \rightarrow \infty} \zeta^{i, N}(t, x)\right) e_{i}$ converges in $\Xi^{*}$. We define

$$
\begin{aligned}
& \zeta(t, x)=\sum_{i=1}^{\infty}\left(\lim _{N \rightarrow \infty} \zeta^{i, N}(t, x)\right) e_{i}, \quad(t, x) \in C, \\
& \zeta(t, x)=0, \quad(t, x) \notin C .
\end{aligned}
$$


Since $Z$ satisfies

$$
Z(\omega, s, t, x)=\sum_{i=1}^{\infty}\left(\lim _{N \rightarrow \infty} Z^{i, N}(\omega, s, t, x)\right) e_{i},
$$

for every $\omega, s, t, x$. From (53), it follows that for every $t, x$, we have $\left(s, X_{s}(\omega, t, x)\right) \in C, P$-a.s., for almost all $s \in[t, \infty)$, and $Z(s, t, x)=\zeta\left(s, X_{s}(t, x)\right) P$-a.s., for a.a. $s \in[t, \infty)$.

We define $v(t, x)=Y(t, t, x)$; since $Y(t, t, x)$ is deterministic, so the map $(t, x) \rightarrow v(t, x)$ can be written as a composition $v(t, x)=\Gamma_{3}\left(\Gamma_{2}\left(t, \Gamma_{1}(t, x)\right)\right)$ with

$$
\begin{gathered}
\Gamma_{1}:[0, \infty) \times \mathscr{C} \longrightarrow L_{\mathscr{P}}^{p}\left(\Omega ; C_{\delta}(R)\right), \\
\Gamma_{1}(t, x)=Y(\cdot, t, x), \\
\Gamma_{2}:[0, \infty) \times L_{\mathscr{P}}^{p}\left(\Omega ; C_{\delta}(R)\right) \longrightarrow L^{p}(\Omega, R), \\
\Gamma_{2}(t, V)=V(t), \\
\Gamma_{3}: L^{p}(\Omega, R) \longrightarrow R, \quad \Gamma_{3} \xi=E \xi .
\end{gathered}
$$

From Theorem 7, it follows that $\Gamma_{1}$ is continuous. By

$$
\begin{aligned}
|V(t)-U(s)|_{L^{p}(\Omega, R)} \leq & |V(t)-V(s)|_{L^{p}(\Omega, R)} \\
& +e^{-\delta p s}|V-U|_{L_{L_{\mathscr{P}}^{p}\left(\Omega ; C_{\delta}(R)\right)}},
\end{aligned}
$$

we have that $\Gamma_{2}$ is continuous. It is clear that $\Gamma_{3}$ is continuous. Then, the map $(t, x) \rightarrow v(t, x)$ is continuous from $[0, \infty) \times \mathscr{C}$ to $R$; therefore, $v(t, x)$ is a Borel measurable function. From uniqueness of the solution of (36), it follows that $Y(s, t, x)=$ $v\left(s, X_{s}(t, x)\right), P$-a.s., for a.a. $s \in[t, \infty)$.

\section{The Fundamental Relation}

Let $(\Omega, \mathscr{F}, P)$ be a given complete probability space with a filtration $\left\{\mathscr{F}_{t}\right\}_{t \geq 0}$ satisfying the usual conditions. $\{W(t), t \geq 0\}$ is a cylindrical Wiener process in $\Xi$ with respect to $\left\{\mathscr{F}_{t}\right\}_{t \geq 0}$. We will say that an $\{\mathscr{F}\}_{t \geq 0}$-predictable process $u$ with values in a given measurable space $(U, \mathcal{U})$ is an admissible control. The function $R:[0, \infty) \times \mathscr{C} \times U \rightarrow \Xi$ is measurable and bounded. We consider the following controlled state equation:

$$
\begin{gathered}
d X^{u}(s)=A X^{u}(s) d s+F\left(s, X_{s}^{u}\right) d s \\
+G\left(s, X_{s}^{u}\right) R\left(s, X_{s}^{u}, u(s)\right) d s+G\left(s, X_{s}^{u}\right) d W(s), \\
s \in[t, \infty), \\
X_{t}^{u}=x .
\end{gathered}
$$

Here, we assume that there exists a mild solution of (58) which will be denoted by $X^{u}(s, t, x)$ or simply by $X^{u}(s)$. We consider a cost function of the form:

$$
J(u)=E \int_{t}^{\infty} e^{-\lambda s} g\left(s, X_{s}^{u}, u(s)\right) d s .
$$

Here, $g$ is function on $[0, \infty) \times \mathscr{C} \times U$ with real values. Our purpose is to minimize the function $J$ over all admissible controls.

We define in a classical way the Hamiltonian function relative to the previous problem; for all $t \in[0, \infty), x \in$ $\mathscr{C}$, and $z \in \Xi^{*}$,

$$
\psi(t, x, z)=\inf \{g(t, x, u)+z R(t, x, u): u \in U\} .
$$

and the corresponding, possibly empty, set of minimizers

$$
\Gamma(t, x, z)=\{u \in U, g(t, x, u)+z R(t, x, u)=\psi(t, x, z)\} .
$$

We are now ready to formulate the assumptions we need.

Hypothesis 3. (i) A, F, and $G$ verify Hypothesis 1.

(ii) $(U, \mathcal{U})$ is a measurable space. The map $g:[0, \infty) \times \mathscr{C} \times$ $U \rightarrow R$ is continuous and satisfies $|g(t, x, u)| \leq K_{g}\left(1+|x|_{C}^{m_{g}}\right)$ for suitable constants $K_{g}>0, m_{g}>0$ and all $x \in \mathscr{C}, u \in$ $U$. The map $R:[0, \infty) \times \mathscr{C} \times U \rightarrow \Xi$ is measurable, and $|R(t, s, u)| \leq L_{R}$ for a suitable constant $K_{R}>0$ and all $x \in$ $\mathscr{C}, u \in U$, and $z \in \Xi^{*}$.

(iii) The Hamiltonian $\psi$ defined in (60) satisfies the requirements of Hypothesis 2 (with $K=R$ ).

(iv) We fix here $p>2$, q and $\delta<0$ satisfying (39) with $\eta=\eta(q)$ and such that $q>m_{g}$.

We are in a position to prove the main result of this section.

Theorem 11. Assume that Hypothesis 3 holds, and suppose that $\lambda$ verifies

$$
\begin{aligned}
\lambda & >\left(-\delta-\mu+\frac{L_{z}^{2}}{2}\right) \vee\left(-\delta+\frac{L_{R}^{2}}{2(p-1)}\right) \\
& \vee\left(\frac{L_{R}^{2} m_{g}}{2\left(q-m_{g}\right)}-\eta(q) m_{g}\right) .
\end{aligned}
$$

Let $v, \zeta$ denote the function in the statement of Theorem 10. Then, for every admissible control $u$ and for the corresponding trajectory $X$ starting at $(t, x)$, one has

$$
\begin{aligned}
J(u)= & v(t, x)+E \int_{t}^{\infty} e^{-\lambda s}\left[-\psi\left(s, X_{s}^{u}, \zeta\left(s, X_{s}^{u}\right)\right)+\zeta\left(s, X_{s}^{u}\right)\right. \\
& \left.\times R\left(s, X_{s}^{u}, u(s)\right)+g\left(s, X_{s}^{u}, u(s)\right)\right] d s .
\end{aligned}
$$

Proof. Consider (58) in the probability space $(\Omega, \mathscr{F}, P)$ with filtration $\left\{\mathscr{F}_{t}\right\}_{t \geq 0}$ and with an $\left\{\mathscr{F}_{t}\right\}_{t \geq 0}$-cylindrical Wiener process $\{W(t), t \geq 0\}$. Let us define

$$
\begin{gathered}
W^{u}(s)=W(s)+\int_{t \wedge s}^{s} R\left(\sigma, X_{\sigma}^{u}, u(\sigma)\right) d \sigma, s \in[0, \infty), \\
\rho(T)=\exp \left(\int_{t}^{T}-R^{*}\left(s, X_{s}^{u}, u(s)\right) d W(s)\right. \\
\left.-\frac{1}{2} \int_{t}^{T}\left|R\left(s, X_{s}^{u}, u(s)\right)\right|^{2} d s\right) .
\end{gathered}
$$


Let $P^{u}$ be the unique probability on $\mathscr{F}_{[0, \infty)}$ such that

$$
\left.P^{u}\right|_{\mathscr{F}_{T}}=\left.\rho(T) P\right|_{\mathscr{F}_{T}} .
$$

We notice that under $P^{u}$, the process $W^{u}$ is a Wiener process. Let us denote by $\left\{\mathscr{F}_{t}^{u}\right\}_{t \geq 0}$ the filtration generated by $W^{u}$, and completed in the usual way. Relatively to $W^{u}$ (58) can be rewritten as

$$
\begin{gathered}
d X^{u}(s)=A X^{u}(s) d s+F\left(s, X_{s}^{u}\right) d s \\
+G\left(s, X_{s}^{u}\right) d W^{u}(s), \quad s \in[t, \infty), \\
X_{t}^{u}=x .
\end{gathered}
$$

In the space $\left(\Omega, \mathscr{F}_{[0, \infty)},\left\{\mathscr{F}_{t}^{u}\right\}_{t \geq 0}, P^{u}\right)$, we consider the following system of forward-backward equations:

$$
\begin{aligned}
& X^{u}(s)=e^{(s-t) A} x(0)+\int_{t}^{s} e^{(s-\sigma) A} F\left(\sigma, X_{\sigma}^{u}\right) d \sigma \\
& +\int_{t}^{s} e^{(s-\sigma) A} G\left(\sigma, X_{\sigma}^{u}\right) d W^{u}(\sigma), \quad s \in[t, \infty), \\
& X_{t}^{u}=x \in \mathscr{C}, \\
& Y^{u}(s)-Y^{u}(T)+\int_{s}^{T} Z^{u}(\sigma) d W^{u}(\sigma)+\lambda \int_{s}^{T} Y^{u}(\sigma) d \sigma \\
& =\int_{s}^{T} \psi\left(\sigma, X_{\sigma}^{u}, Z^{u}(\sigma)\right) d \sigma, \quad 0 \leq s \leq T<\infty .
\end{aligned}
$$

Applying the Itô formula to $e^{-\lambda s} Y^{u}(s)$ and writing the backward equation in (67) with respect to the process $W$, we get

$$
\begin{aligned}
Y^{u}(s)+ & \int_{s}^{T} e^{-\lambda \sigma} Z^{u}(\sigma) d W(\sigma) \\
= & \int_{s}^{T} e^{-\lambda \sigma}\left[\psi\left(\sigma, X_{\sigma}^{u}, Z^{u}(\sigma)\right)\right. \\
& \left.-Z^{u}(\sigma) R\left(\sigma, X_{\sigma}^{u}, u(\sigma)\right)\right] d \sigma \\
& +e^{-\lambda T} Y^{u}(T) .
\end{aligned}
$$

Recalling that $R$ is bounded, we get, for all $r \geq 1$ and some constant $C$,

$$
\begin{array}{r}
E^{u}\left[\rho(T)^{-r}\right]=E^{u}\left[\operatorname { e x p } r \left(\int_{t}^{T} R^{*}\left(s, X_{s}^{u}, u(s)\right) d W^{u}(s)\right.\right. \\
\left.\left.-\frac{1}{2} \int_{t}^{T}\left|R\left(s, X_{s}^{u}, u(s)\right)\right|^{2} d s\right)\right]
\end{array}
$$

$$
\begin{aligned}
& =E^{u}\left[\operatorname { e x p } \left(\int_{t}^{T} r R^{*}\left(s, X_{s}^{u}, u(s)\right) d W^{u}(s)\right.\right. \\
& \left.\quad-\frac{1}{2} \int_{t}^{T} r^{2}\left|R\left(s, X_{s}^{u}, u(s)\right)\right|^{2} d s\right) \\
& \left.\quad \times \exp \frac{r(r-1)}{2} \int_{t}^{T}\left|R\left(s, X_{s}^{u}, u(s)\right)\right|^{2} d s\right] \\
& \leq e^{(1 / 2) r(r-1) T L_{R}^{2}} E^{u} \\
& \quad \times \exp \left(\int_{t}^{T} 2 R^{*}\left(s, X_{s}^{u}, u(s)\right) d W^{u}(s)\right. \\
& \left.\quad-\frac{1}{2} \int_{t}^{T} 4\left|R\left(s, X_{s}^{u}, u(s)\right)\right|^{2} d s\right) \\
& =e^{(1 / 2) r(r-1) T L_{R}^{2}} .
\end{aligned}
$$

It follows that

$$
\begin{aligned}
E\left(\int_{t}^{T}\left|e^{-\lambda s} Z^{u}(s)\right|^{2} d s\right)^{1 / 2} & =E^{u}\left[\left(\int_{t}^{T}\left|e^{-\lambda s} Z^{u}(s)\right|^{2} d s\right)^{1 / 2} \rho^{-1}\right] \\
\leq & \left(E^{u} \int_{t}^{T}\left|e^{-\lambda s} Z^{u}(s)\right|^{2} d s\right)^{1 / 2} \\
& \times\left(E^{u} \rho^{-2}\right)^{1 / 2}<\infty .
\end{aligned}
$$

We conclude that the stochastic integral in (68) has zero expectation. If we set $s=t$ in (68) and we take expectation with respect to $P$, we obtain

$$
\begin{aligned}
e^{-\lambda T} E Y^{u}(T)-Y^{u}(t) & \\
=E \int_{t}^{T} e^{-\lambda \sigma}[ & -\psi\left(\sigma, X_{\sigma}^{u}, Z^{u}(\sigma)\right) \\
& \left.+Z^{u}(\sigma) R\left(\sigma, X_{\sigma}^{u}, u(\sigma)\right)\right] d \sigma .
\end{aligned}
$$

By Theorem 7, $Y^{u}(\cdot, t, x) \in L_{\mathscr{P}}^{p}\left(\Omega ; C_{\delta}(R)\right)$, so that

$$
E^{u}|Y(T, t, x)|^{p} \leq C \exp (-p \delta T) .
$$

By the Hölder inequality, we have that for suitable constant $C>0$,

$$
\begin{aligned}
E|Y(T, t, x)| & =E^{u}\left(\rho^{-1}(T)|Y(T, t, x)|\right) \\
& \leq E\left(\rho^{-p /(p-1)}\right)^{(p-1) / p} E\left(|Y(T, t, x)|^{p}\right)^{1 / p} \\
& \leq C e^{\left.\left(\left(L_{R}^{2} / 2(p-1)\right)-\delta\right)\right) T} .
\end{aligned}
$$

From Theorem 2, we obtain $E^{u} \sup _{s \geq t} e^{\eta q s}\left|X_{s}^{u}\right|^{q}<\infty$; by the similar process, we get that

$$
E\left|X_{T}^{u}\right|^{m_{g}} \leq C e^{\left(L_{R}^{2} m_{g}\left(2 q-2 m_{g}\right)^{-1}-\eta(q) m_{g}\right) T},
$$


for suitable constant $C>0$ and

$$
E \int_{t}^{\infty} e^{-\lambda \sigma}\left|g\left(\sigma, X_{\sigma}^{u}, u(\sigma)\right)\right| d \sigma<\infty .
$$

Since $Y^{u}(t, t, x)=v(t, x)$ and $Z^{u}(s, t, x)=\zeta\left(s, X_{s}^{u}(t, x)\right), P$ a.s., for a.a. $s \in[t, \infty)$, we have that

$$
\begin{aligned}
& e^{-\lambda T} E Y^{u}(T)-v(t, x) \\
&=E \int_{t}^{T} e^{-\lambda \sigma}\left[-\psi\left(\sigma, X_{\sigma}^{u}, \zeta\left(\sigma, X_{\sigma}^{u}\right)\right)\right. \\
&\left.+\zeta\left(\sigma, X_{\sigma}^{u}\right) R\left(\sigma, X_{\sigma}^{u}, u(\sigma)\right)\right] d \sigma .
\end{aligned}
$$

Thus, adding and subtracting $E \int_{t}^{\infty} e^{-\lambda \sigma} g\left(\sigma, X_{\sigma}^{u}, u(\sigma)\right) d \sigma$ and letting $T \rightarrow \infty$, we conclude that

$$
\begin{aligned}
J(u)=v(t, x) & \\
+E \int_{t}^{\infty} e^{-\lambda s}[ & -\psi\left(s, X_{s}^{u}, \zeta\left(s, X_{s}^{u}\right)\right)+\zeta\left(s, X_{s}^{u}\right) R \\
& \left.\times\left(s, X_{s}^{u}, u(s)\right)+g\left(s, X_{s}^{u}, u(s)\right)\right] d s .
\end{aligned}
$$

The proof is finished.

We immediately deduce the following consequences.

Theorem 12. Let $t \in[0, \infty)$ and $x \in \mathscr{C}$ be fixed, assume that the set-valued map $\Gamma$ has nonempty values and it admits a measurable selection $\Gamma_{0}:[0, \infty) \times \mathscr{C} \times \Xi^{*} \rightarrow U$, and assume that a control $u(\cdot)$ satisfies

$$
\begin{aligned}
& u(s)=\Gamma_{0}\left(s, X_{s}^{u}, \zeta\left(s, X_{s}^{u}\right)\right), \\
& P \text {-a.s., for almost every } s \in[t, \infty) .
\end{aligned}
$$

Then, $J(t, x, u)=v(t, x)$, and the pair $(u(\cdot), X)$ is optimal for the control problem starting from $x$ at time $t$.

Such a control can be shown to exist if there exists a solution for the so-called closed-loop equation as follows:

$$
\begin{gathered}
d X(s)=A X(s) d s+F\left(s, X_{s}\right) d s+G\left(s, X_{s}\right) \\
\times\left(R\left(s, X_{s}, \Gamma_{0}\left(s, X_{s}, \zeta\left(s, X_{s}\right)\right)\right) d s+d W(s)\right), \\
s \in[t, \infty), \\
X_{t}=x,
\end{gathered}
$$

since in this case, we can define an optimal control setting

$$
u(s)=\Gamma_{0}\left(s, X_{s}, \zeta\left(s, X_{s}\right)\right) .
$$

However, under the present assumptions, we cannot guarantee that the closed-loop equation has a solution in the mild sense. To circumvent this difficulty, we will revert to a weak formulation of the optimal control problem.

\section{Existence of Optimal Control}

We formulate the optimal control problem in the weak sense following the approach of [31]. The main advantage is that we will be able to solve the closed-loop equation in a weak sense, and, hence, to find an optimal control, even if the feedback law is nonsmooth.

We call $\left(\Omega, \mathscr{F},\left\{\mathscr{F}_{t}\right\}_{t \geq 0}, P, W\right)$ an admissible setup, if $\left(\Omega, \mathscr{F},\left\{\mathscr{F}_{t}\right\}_{t \geq 0}, P\right)$ is a filtered probability space satisfying the usual conditions, and $W$ is a cylindrical $P$-Wiener process with values in $\Xi$, with respect to the filtration $\left\{\mathscr{F}_{t}\right\}_{t \geq 0}$.

By an admissible control system, we mean $(\Omega, \mathscr{F}$, $\left.\left\{\mathscr{F}_{t}\right\}_{t \geq 0}, P, W, u, X^{u}\right)$, where $\left(\Omega, \mathscr{F},\left\{\mathscr{F}_{t}\right\}_{t \geq 0}, P, W\right)$ is an admissible setup, $u$ is an $\mathscr{F}_{t}$-predictable process with values in $U$, and $X^{u}$ is a mild solution of (58). An admissible control system will be briefly denoted by $\left(W, u, X^{u}\right)$ in the following. Our purpose is to minimize the cost functional

$$
J(u)=E \int_{t}^{\infty} e^{-\lambda s} g\left(s, X_{s}^{u}, u(s)\right) d s
$$

over all the admissible control system.

Our main result in this section is based on the solvability of the closed-loop equation

$$
\begin{gathered}
d X(s)=A X(s) d s+F\left(s, X_{s}\right) d s+G\left(s, X_{s}\right) \\
\times\left(R\left(s, X_{s}, \Gamma_{0}\left(s, X_{s}, \zeta\left(s, X_{s}\right)\right)\right) d s+d W(s)\right), \\
s \in[t, \infty), \\
X_{t}=x .
\end{gathered}
$$

In the following sense, we say that $X$ is a weak solution of (82) if there exists an admissible setup $\left(\Omega, \mathscr{F},\left\{\mathscr{F}_{t}\right\}_{t \geq 0}, P, W\right)$ and an $\mathscr{F}_{t}$-adapted continuous process $X(t)$ with values in $H$, which solves the equation in the mild sense; namely, $P$-a.s.,

$$
\begin{aligned}
& X(s)= e^{(s-t) A} x(0)+\int_{t}^{s} e^{(s-\sigma) A} F\left(\sigma, X_{\sigma}\right) d \sigma \\
&+\int_{t}^{s} e^{(s-\sigma) A} G\left(\sigma, X_{\sigma}\right) R \\
& \times\left(\sigma, X_{\sigma}, \Gamma_{0}\left(\sigma, X_{\sigma}, \zeta\left(\sigma, X_{\sigma}\right)\right)\right) d \sigma \\
&+\int_{t}^{s} e^{(s-\sigma) A} G\left(\sigma, X_{\sigma}\right) d W_{\sigma}, \quad s \in[t, \infty), \\
& X_{t}=x .
\end{aligned}
$$

Theorem 13. Assume that Hypothesis 3 holds. Then, there exists a weak solution of the closed-loop equation (82) which is unique in law. 
Proof (uniqueness). Let $X$ be a weak solution of (82) in an admissible setup $\left(\Omega, \mathscr{F},\left\{\mathscr{F}_{t}\right\}_{t \geq 0}, P, W\right)$. We define

$$
\begin{aligned}
\rho(T)=\exp \left(\int_{t}^{T}-R^{*}\left(\sigma, X_{\sigma}, \Gamma_{0}\left(\sigma, X_{\sigma}, \zeta\left(\sigma, X_{\sigma}\right)\right)\right) d W(\sigma)\right. \\
\left.-\frac{1}{2} \int_{t}^{T}\left|R\left(\sigma, X_{\sigma}, \Gamma_{0}\left(\sigma, X_{\sigma}, \zeta\left(\sigma, X_{\sigma}\right)\right)\right)\right|^{2} d \sigma\right) .
\end{aligned}
$$

Since $R$ is bounded, the Girsanov theorem ensures that there exists a probability measure $P^{0}$ such that the process

$$
\begin{array}{r}
W^{0}(s)=W(s)+\int_{t \wedge s}^{s} R\left(\sigma, X_{\sigma}, \Gamma_{0}\left(\sigma, X_{\sigma}, \zeta\left(\sigma, X_{\sigma}\right)\right)\right) d \sigma, \\
s \in[0, \infty),
\end{array}
$$

is a $P^{0}$-Wiener process and

$$
\left.P^{0}\right|_{\mathscr{F}_{T}}=\left.\rho(T) P\right|_{\mathscr{F}_{T}} .
$$

Let us denote by $\left\{\mathscr{F}_{t}^{0}\right\}_{t \geq 0}$ the filtration generated by $W^{0}$ and completed in the usual way. In $\left(\Omega, \mathscr{F}_{[0, \infty)},\left\{\mathscr{F}_{t}^{0}\right\}_{t \geq 0}, P^{0}\right), X$ is a mild solution of

$$
\begin{gathered}
d X(s)=A X(s) d s+F\left(t, X_{s}\right) d s \\
+G\left(s, X_{s}\right) d W^{0}(s), \quad s \in[t, \infty) \\
X_{t}=x, \\
\rho(T)=\exp \left(\int_{t}^{T}-R^{*}\left(\sigma, X_{\sigma}, \Gamma_{0}\left(\sigma, X_{\sigma}, \zeta\left(\sigma, X_{\sigma}\right)\right) d W^{0}(\sigma)\right.\right. \\
\left.+\frac{1}{2} \int_{t}^{T}\left|R\left(\sigma, X_{\sigma}, \Gamma_{0}\left(\sigma, X_{\sigma}, \zeta\left(\sigma, X_{\sigma}\right)\right)\right)\right|^{2} d \sigma\right) .
\end{gathered}
$$

By Hypothesis 3, the joint law of $X$ and $W^{0}$ is uniquely determined by $A, F, G$, and $x$. Taking into account the last displayed formula, we conclude that the joint law of $X$ and $\rho(T)$ under $P^{0}$ is also uniquely determined, and consequently so is the law of $X$ under $P$. This completes the proof of the uniqueness part.

Proof (existence). Let $(\Omega, \mathscr{F}, P)$ be a given complete probability space. $\{W(t), t \geq 0\}$ is a cylindrical Wiener process on $(\Omega, \mathscr{F}, P)$ with values in $\Xi$, and $\left\{\mathscr{F}_{t}\right\}_{t \geq 0}$ is the natural filtration of $\{W(t), t \geq 0\}$, augmented with the family of $P$-null sets. Let $X(\cdot)$ be the mild solution of

$$
\begin{gathered}
d X(s)=A X(s) d s+F\left(s, X_{s}\right) d s \\
+G\left(s, X_{s}\right) d W(s), \quad s \in[t, \infty), \\
\quad X_{t}=x,
\end{gathered}
$$

and by the Girsanov theorem, let $P^{1}$ be the probability on $\Omega$ under which

$$
W^{1}(s)=W(s)-\int_{t \wedge s}^{s} R\left(\sigma, X_{\sigma}, \Gamma_{0}\left(\sigma, X_{\sigma}, \zeta\left(\sigma, X_{\sigma}\right)\right)\right) d \sigma
$$

is a Wiener process (notice that $R$ is bounded). Then, $X$ is the weak solution of $(82)$ relatively to the probability $P^{1}$ and the Wiener process $W^{1}$.

Now, we can state the main result of this section.

Corollary 14. Assume that Hypothesis 3 holds true and $\lambda$ verifies (62) Also, assume that the set-valued map $\Gamma$ has nonempty values and it admits a measurable selection $\Gamma_{0}$ : $[0, \infty) \times \mathscr{C} \times \Xi^{*} \rightarrow U$. Then, for every $t \in[0, \infty)$ and $x \in \mathscr{C}$ and for all admissible control system $\left(W, u, X^{u}\right)$, one has

$$
J(u, t, x) \geq v(t, x),
$$

and the equality holds if

$$
\begin{aligned}
& u(s)=\Gamma_{0}\left(s, X_{s}^{u}, \zeta\left(s, X_{s}^{u}\right)\right), \\
& P-\text { a.s. for almost every } s \in[t, \infty) .
\end{aligned}
$$

Moreover, from Theorem 13, it follows that the closedloop equation (82) admits a weak solution $(\Omega, \mathscr{F}$, $\left.\left\{\mathscr{F}_{t}\right\}_{t \geq 0}, P, W, X\right)$ which is unique in law, and setting

$$
u(s)=\Gamma_{0}\left(s, X_{s}, \zeta\left(s, X_{s}\right)\right),
$$

we obtain an optimal admissible control system $(W, u, X)$.

\section{Applications}

In this section, we present a simple application of the previous results. We consider the stochastic delay partial differential equation in the bounded domain $B \subset R^{n}$ with smooth boundary $\partial B$ as follows:

$$
\begin{aligned}
& d z^{u}(t, \xi)= \Delta z^{u}(t, \xi) d t+f\left(t, z_{t}^{u}(\xi)\right) d t \\
&+\sum_{i=1}^{d} g_{i}\left(t, z_{t}^{u}(\xi)\right)\left[r^{i}(\xi) u^{i}(t) d t+d W^{i}(t)\right], \\
& z_{0}^{u}(\theta, \xi)= x(\theta, \xi), \quad \xi \in B, \theta \in[-1,0], \\
& z^{u}(t, \xi)=0, \quad t \in[0, \infty), \quad \xi \in \partial B .
\end{aligned}
$$

Here, $W=\left(W^{1}, W^{2}, \ldots, W^{d}\right)$ is a standard Wiener process in $R^{d}$, and the functions $f:[0,+\infty) \times C([-1,0], R) \rightarrow R$ and $g_{i}:[0,+\infty) \times C([-1,0], R) \rightarrow R$ are Lipschitz continuous and bounded. Setting $U$ as a bounded subset of $R^{d}, \Xi=R^{d}$, $H=L^{2}(B)$, and $x \in C([-1,0], H)$. We define $F$ and $G$ as following:

$$
\begin{gathered}
F(t, x)(\xi)=f(t, x(\xi)), \\
(G(t, x) z)(\xi)=\sum_{i=1}^{d} g_{i}(t, x(\xi)) z^{i}(\xi), \\
\xi \in B, \quad x \in C([-1,0], H), \quad z \in L(\Xi, H),
\end{gathered}
$$

and let $A$ denote the Laplace operator $\Delta$ in $L^{2}(B)$; with domain $W^{2,2}(B) \bigcap W_{0}^{1,2}(B)$ then, (94) has the form (58) and Hypothesis 1 holds. 
Let us consider the optimal control problem associated with the cost

$$
J(u)=E \int_{0}^{\infty} e^{-\lambda t}\left[\int_{B} \sigma\left(\xi, z_{t}^{u}(\xi)\right) d \xi+u^{2}(t)\right] d t
$$

where $\lambda$ verifies (62) and $\sigma: C([-1,0], R) \times U \rightarrow[0, \infty)$ is a bounded measurable function. Define $g: C([-1,0], H) \times$ $U \rightarrow[0, \infty)$ and $R: C([-1,0], H) \times U \rightarrow \quad \Xi$ by $g(y, u)=\int_{B} \sigma(t, y(\xi), u) d \xi+u^{2}$ and $R(y, u)=\left(\int_{B} r^{1}(\xi) u^{1} d \xi\right.$, $\left.\int_{B} r^{2}(\xi) u^{2} d \xi, \ldots, \int_{B} r^{d}(\xi) u^{d} d \xi\right)$ for $y \in C([-1,0], H), u=$ $\left(u^{1}, u^{2}, \ldots, u^{d}\right) \in U$, respectively. It can be easily verified that Hypothesis 3 holds true, and the set-valued map $\Gamma$ has nonempty values and it admits a measurable selection $\Gamma_{0}$ : $[0, \infty) \times \mathscr{C} \times \Xi^{*} \rightarrow U$. Then, the closed-loop equation (82) admits a weak solution $\left(\Omega, \mathscr{F},\left\{\mathscr{F}_{t}\right\}_{t \geq 0}, P, W, u, z .(\cdot)\right)$, and setting

$$
u(s)=\Gamma_{0}\left(s, z_{s}(\cdot), \zeta\left(s, z_{s}(\cdot)\right)\right)
$$

we obtain an optimal admissible control system $(W, u, z(\cdot))$.

\section{References}

[1] J. Bismut, "On optimal control of linear stochastic equations with a linear-quadratic criterion," SIAM Journal on Control and Optimization, vol. 15, no. 3, pp. 1-4, 1977.

[2] N. Nagase, "On the existence of optimal control for controlled stochastic partial differential equations," Nagoya Mathematics Journal, vol. 115, pp. 73-85, 1989.

[3] N. El Karoui, D. Huu Nguyen, and M. Jeanblanc-Piqué, "Compactification methods in the control of degenerate diffusions," Stochastics, vol. 20, pp. 169-219, 1987.

[4] M. Nisio, "Optimal control for stochastic partial differential equations and viscosity solutions of Bellman equations," Nagoya Mathematics Journal, vol. 123, pp. 13-37, 1991.

[5] M. Nisio, "On sensitive control for stochastic partial differential equations," in Stochastic Analysis on Infinite Dimensional Spaces Proceedings of the U.S. Japan Bilateral Seminar, H. Kunita et al., Ed., vol. 310 of Pitman Research Notes Mathematical Series, pp. 231-241, Longman Scientific and Technical, Baton Rouge, La, USA, January 1994.

[6] R. Buckdahn and A. Raşcanu, "On the existence of stochastic optimal control of distributed state system," Nonlinear Analysis, Theory, Methods and Applications, vol. 52, no. 4, pp. 1153-1184, 2003.

[7] V. Barbu and G. Da Prato, Equations in Hilbert Spaces, vol. 86 of Pitman Research Notes in Mathematics, Pitman, 1983.

[8] P. Cannarsa and G. Da Prato, "Second-order Hamilton-Jacobi equations in infinite dimensions," SIAM Journal on Control and Optimization, vol. 29, no. 2, pp. 474-492, 1991.

[9] P. Cannarsa and G. Da Prato, "Direct solution of a second-order Hamilton-Jacobi equations in Hilbert spaces," in Stochastic Partial Differential Equations and Applications, G. Da Prato and L. Tubaro, Eds., vol. 268 of Pitman Research Notes in Mathematics, Pitman, 1992.

[10] F. Gozzi, "Regularity of solutions of second order HamiltonJacobi equations and application to a control problem," Communications in Partial Differential Equations, vol. 20, pp. 775-826, 1995.
[11] F. Gozzi, "Global regular solutions of second order HamiltonJacobi equations in Hilbert spaces with locally Lipschitz nonlinearities," Journal of Mathematical Analysis and Applications, vol. 198, no. 2, pp. 399-443, 1996.

[12] E. Pardoux and S. G. Peng, "Adapted solution of a backward stochastic differential equation," Systems and Control Letters, vol. 14, no. 1, pp. 55-61, 1990.

[13] N. El Karoui and L. Mazliak, Eds., Backward Stochastic Differential Equations, vol. 364 of Pitman Research Notes in Mathematics Series, Longman, 1997.

[14] E. Pardoux and BSDEs, "weak convergence and homogeneization of semilinear PDEs," in Non- Linear Analysis, Differential Equations and Control, F. H. Clarke and R. J. Stern, Eds., pp. 503-549, Kluwer, Dordrecht, The Netherlands, 1999.

[15] S. Peng, "A generalized dynamic programming principle and Hamilton-Jacobi-Bellman equation," Stochastics and Stochastics Reports, vol. 38, pp. 119-134, 1992.

[16] N. E. Karoui, S. Peng, and M. C. Quenez, "Backward stochastic differential equations in finance," Mathematical Finance, vol. 7, no. 1, pp. 1-71, 1997.

[17] S. Hamad $\mu$ ene and J. P. Lepeltier, "Backward equations, stochastic control and zero-sum stochastic differential games," Stochastics and Stochastics Reports, vol. 54, pp. 221-231, 1995.

[18] N. El-Karoui and S. Hamadène, "BSDEs and risk-sensitive control, zero-sum and nonzero-sum game problems of stochastic functional differential equations," Stochastic Processes and their Applications, vol. 107, no. 1, pp. 145-169, 2003.

[19] M. Fuhrman and G. Tessiture, "Existence of optimal stochastic controls and global solutions of forward-backward stochastic differential equations," SIAM Journal on Control and Optimization, vol. 43, no. 3, pp. 813-830, 2005.

[20] M. Fuhrman, Y. Hu, and G. Tessitore, "On a class of stochastic optimal control problems related to bsdes with quadratic growth," SIAM Journal on Control and Optimization, vol. 45, no. 4, pp. 1279-1296, 2006.

[21] M. Fuhrman and G. Tessitore, "Nonlinear kolmogorov equations in infinite dimensional spaces: the backward stochastic differential equations approach and applications to optimal control," Annals of Probability, vol. 30, no. 3, pp. 1397-1465, 2002.

[22] F. Masiero, "Semilinear kolmogorov equations and applications to stochastic optimal control," Applied Mathematics and Optimization, vol. 51, no. 1, pp. 201-250, 2005.

[23] M. Fuhrman, F. Masiero, and G. Tessitore, "Stochastic equations with delay: optimal control via BSDEs and regular solutions of Hamilton-jacobi-bellman equations," SIAM Journal on Control and Optimization, vol. 48, no. 7, pp. 4624-4651, 2010.

[24] M. Fuhrman and G. Tessiture, "Infinite horizon backward stochastic differential equations and elliptic equations in hilbert spaces," Annals of Probability, vol. 32, no. 1, pp. 607-660, 2004.

[25] F. Masiero, "Infinite horizon stochastic optimal control problems with degenerate noise and elliptic equations in Hilbert spaces," Applied Mathematics and Optimization, vol. 55, no. 3, pp. 285-326, 2007.

[26] M. Fuhrman, "A class of stochastic optimal control problems in Hilbert spaces: BSDEs and optimal control laws, state constraints, conditioned processes," Stochastic Processes and their Applications, vol. 108, no. 2, pp. 263-298, 2003.

[27] F. Masiero, "Stochastic optimal control problems and parabolic equations in banach spaces," SIAM Journal on Control and Optimization, vol. 47, no. 1, pp. 251-300, 2008.

[28] G. Da Prato and J. Zabczyk, Ergodicity For Infinite-Dimensional Systems, Cambridge University Press, 1996. 
[29] J. Zabczyk, "Parabolic equations on Hilbert spaces," in StochaStic PDE'S and Kolmogorov Equations in Infinite Dimensions, vol. 1715 of Lecture Notes in Math, pp. 117-213, Springer, Berlin, Germany, 1999.

[30] G. Da Prato and J. Zabczyk, Stochstic Equations in Infinite Dimensions, Cambridge University Press, 1992.

[31] W. H. Fleming and H. M. Soner, Controlled Markov Processes and Viscosity Solutions, vol. 25 of Applications of Mathematics, Springer, New York, NY, USA, 1993. 


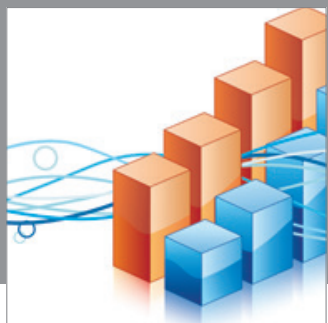

Advances in

Operations Research

mansans

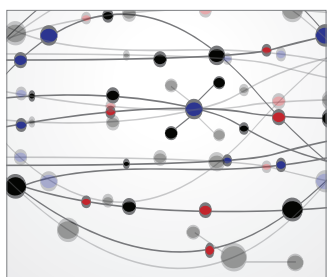

The Scientific World Journal
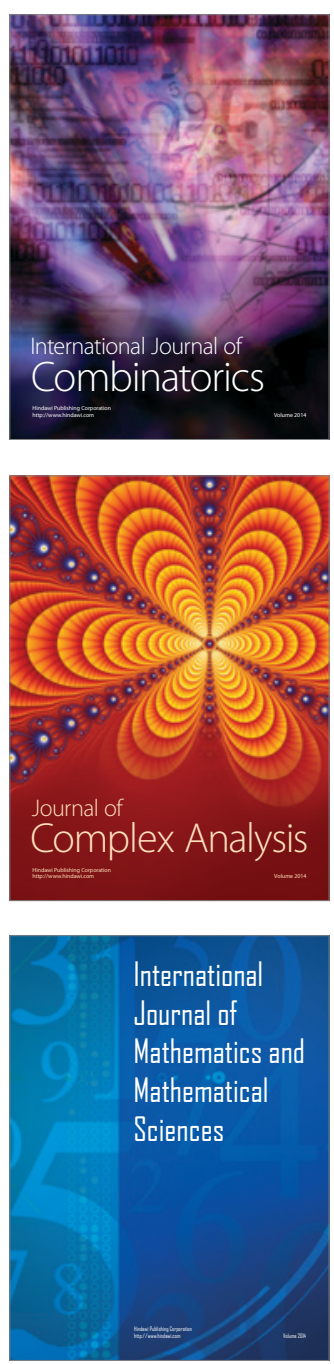
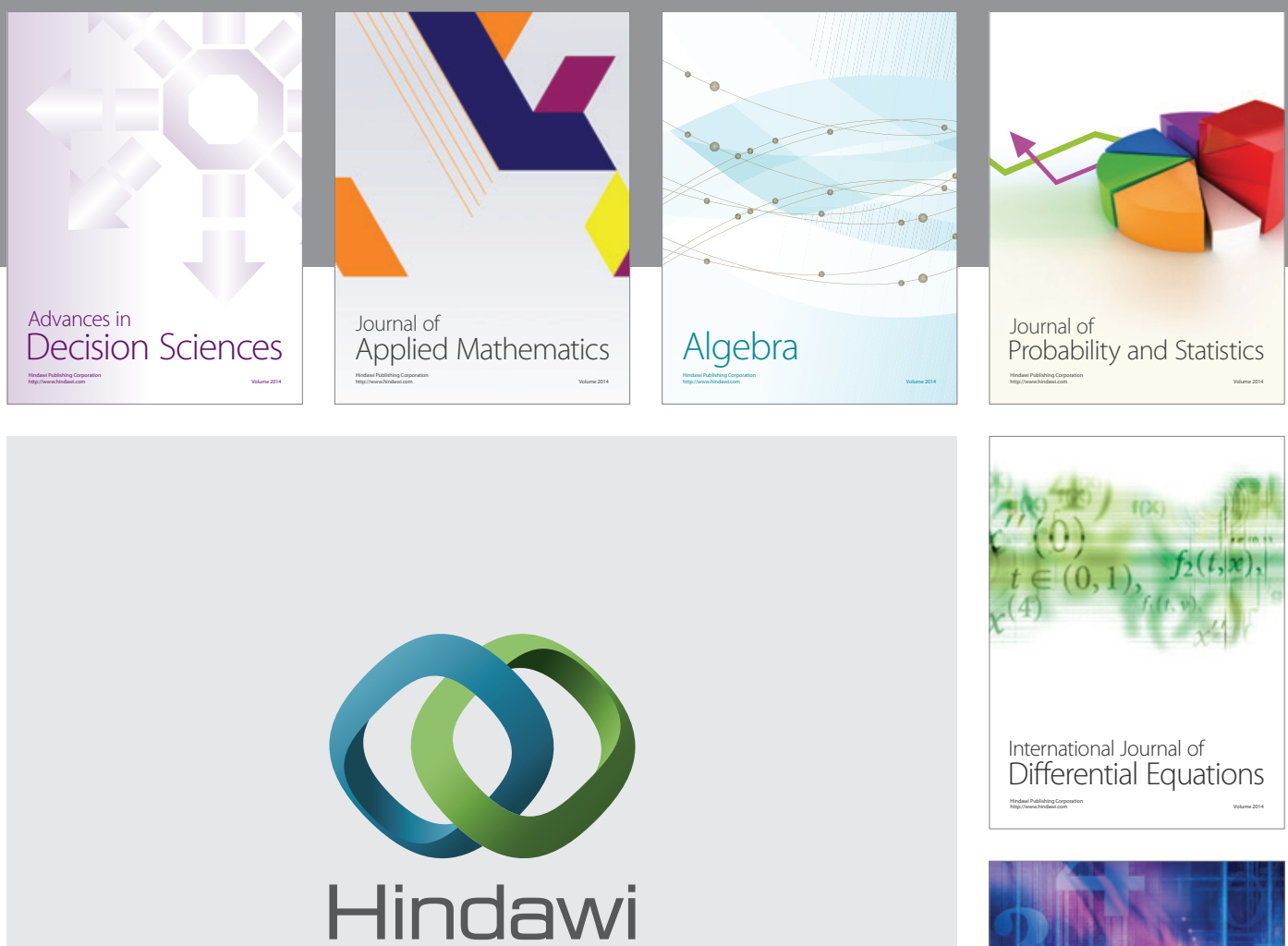

Submit your manuscripts at http://www.hindawi.com
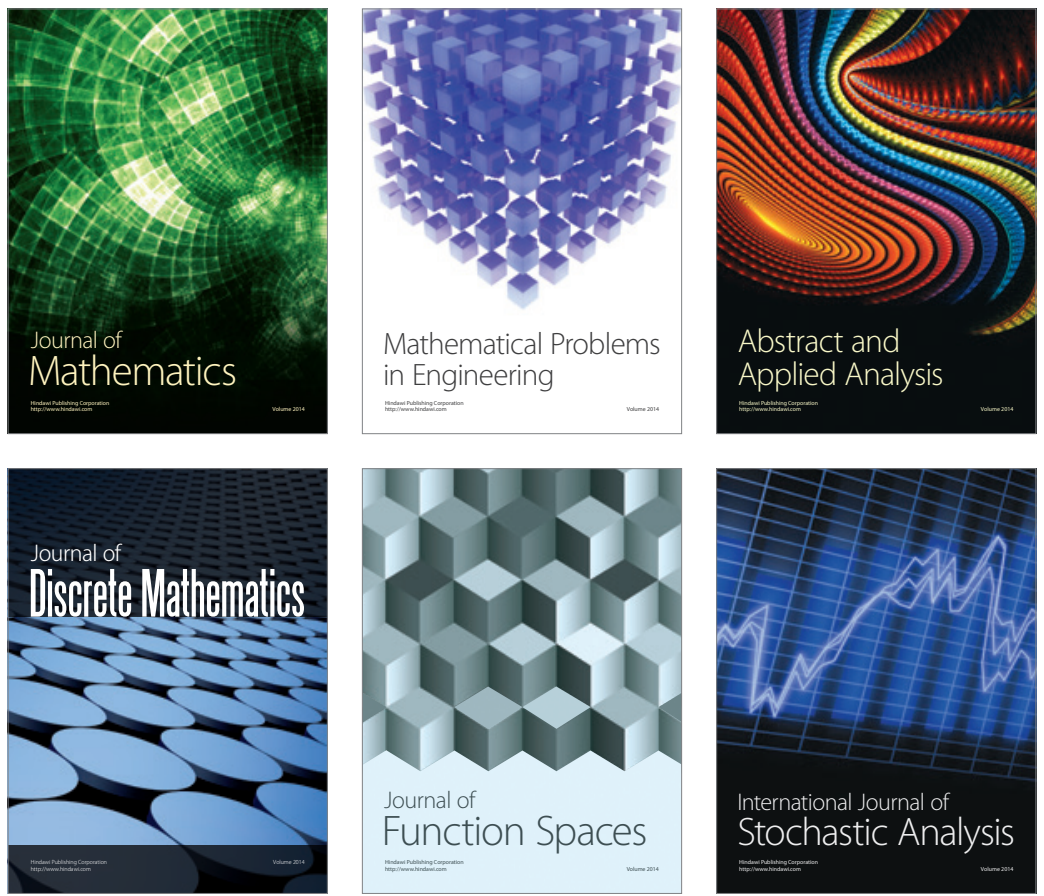

Journal of

Function Spaces

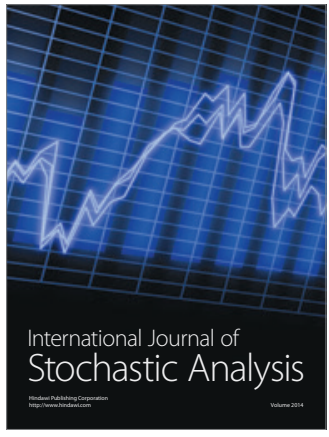

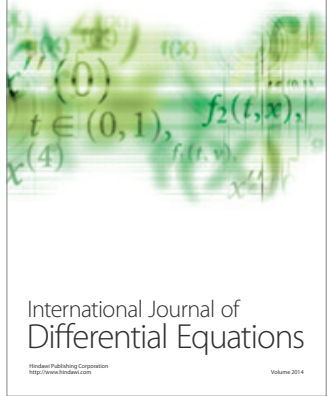
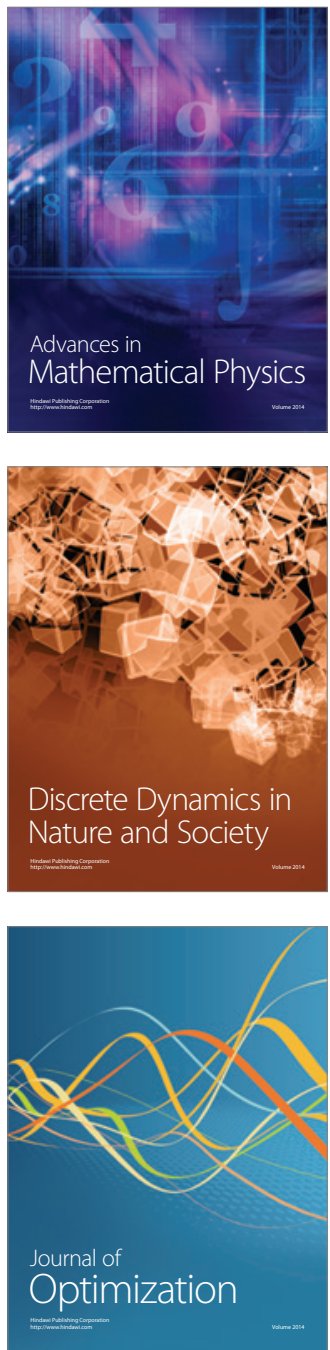